\title{
Evolutionary Conservation of Vertebrate Blood-Brain Barrier Chemoprotective Mechanisms in Drosophila
}

\author{
Fahima Mayer, ${ }^{1 *}$ Nasima Mayer, ${ }^{1 *}$ Leslie Chinn, ${ }^{2}$ Robert L. Pinsonneault, ${ }^{1}$ Deanna Kroetz, ${ }^{2}$ and Roland J. Bainton ${ }^{1}$ \\ ${ }^{1}$ Department of Anesthesia and Perioperative Care, San Francisco General Hospital, and ${ }^{2}$ Department of Pharmaceutical Chemistry, University of California \\ at San Francisco, San Francisco, California 94143
}

Pharmacologic remedy of many brain diseases is difficult because of the powerful drug exclusion properties of the blood-brain barrier (BBB). Chemical isolation of the vertebrate brain is achieved through the highly integrated, anatomically compact and functionally overlapping chemical isolation processes of the BBB. These include functions that need to be coordinated between tight diffusion junctions and unidirectionally acting xenobiotic transporters. Understanding of many of these processes has been hampered, because they are not well mimicked by ex vivo models of the BBB and have been experimentally difficult and expensive to disentangle in intact rodent models. Here we show that the Drosophila melanogaster $(\mathrm{Dm})$ humoral/CNS barrier conserves the xenobiotic exclusion properties found in the vertebrate vascular endothelium. We characterize a fly ATP binding cassette (ABC) transporter, Mdr65, that functions similarly to mammalian xenobiotic BBB transporters and show that varying its levels solely in the $\mathrm{Dm}$ BBB changes the inherent sensitivity of the barrier to cytotoxic pharmaceuticals. Furthermore, we demonstrate orthologous function between Mdr65 and vertebrate $\mathrm{ABC}$ transporters by rescuing chemical protection of the $\mathrm{Dm}$ brain with human MDR1/Pgp. These data indicate that the ancient origins of CNS chemoprotection extend to both conserved molecular means and functionally analogous anatomic spaces that together promote CNS selective drug partition. Thus, Dm presents an experimentally tractable system for analyzing physiological properties of the $\mathrm{BBB}$ in an intact organism.

\section{Introduction}

In vertebrates, a physically separate blood-brain barrier (BBB), primarily engineered into the single-cell layer vascular endothelium (VE), provides an obstacle to chemical attack. At this interface, strong selective pressures have produced the integration of at least two very different cell biologic mechanisms to prevent free movement of small molecules between the humoral and CNS interstitial compartments. (Abbott, 2005; Daneman and Barres, 2005; Neuwelt et al., 2008; Zlokovic, 2008). BBB VE cells impede the traffic of drugs by virtue of specialized lateral junction components, including tight junctions, and asymmetrically arrayed ATP binding cassette $(\mathrm{ABC})$ transporters. Tight junctions prevent paracellular diffusion of charged molecules, and asymmetrically arrayed transporters actively expel lipophilic molecules back into the humoral space (Löscher and Potschka, 2005b). Together, these complimentary systems prevent the majority of xenobiotics from acting on vertebrate nervous tissue (Pardridge, 2005a,b). Although in vivo and in vitro BBB models have confirmed the

\footnotetext{
Received Nov. 19, 2008; revised Jan. 27, 2009; accepted Feb. 5, 2009.

This work was supported by National Institutes of Health Grant GM081863. We thank Nebiyu Tegegn, Kelly Komachi, Owen Hughes, Laura Mitic, Adrian Rothenfluh, and Kaveh Ashrafi for many helpful comments and editing We thank Reed Kelso for his technical assistance early in this work. We are very grateful to Ron Vale, Peter Walter, Eric Griffis, Nico Sturrman, and Toby Walther for assistance in confocal microscopy. We thank Susan Johns for comparative genomic analysis. We also thank the Kenyon, Blackburn, Fung, and $0^{\prime}$ Farrell laboratories for being super neighbors and kind associates.

*F.M. and N.M. contributed equally to this work.

Correspondence should be addressed to Roland J. Bainton, Box 2200, Mission Bay/Genetech Hall S312C, University of California, San Francisco, San Francisco, CA 94158-2517. E-mail: baintonr@anesthesia.ucsf.edu. DOI:10.1523/JNEUROSCI.5564-08.2009

Copyright $\odot 2009$ Society for Neuroscience $\quad$ 0270-6474/09/293538-13\$15.00/0
}

importance of these two components (Schinkel et al., 1997; Nitta et al., 2003), substantial limitations hinder progress (Garberg et al., 2005). A powerful BBB model system should combine molecular genetic, genomic, chemical biology, and integrative physiology tools to probe CNS-specific chemoprotective physiology. For this, we turned to Drosophila melanogaster $(\mathrm{Dm})$ and asked what aspects of BBB physiology can be modeled in an invertebrate.

Insects also possess protective neural barriers, but they differ anatomically from vertebrates (see Fig. 1). Dm has an open circulatory system that is separated from the CNS by a thin layer of glially derived epithelial cells (Treherne, 1972; Carlson et al., 2000; Stork et al., 2008), making the Dm humoral/CNS interface topologically much simpler than the vertebrate BBB. However, on a cellular level, the vertebrate and insect BBBs share many common features. In particular, one specific cell layer of the Dm $\mathrm{BBB}$, the subperineural glia (SPG), possesses elaborate laterally localized homotypic junctional complexes, or pleated septate junctions, that create a tight barrier to paracellular diffusion (Edwards et al., 1993; Tepass and Hartenstein, 1994). The Dm proteins that make up the pleated septate junctions are nearly identical to the vertebrate proteins that compose the tight junctions (Wu and Beitel, 2004; Banerjee et al., 2006). Furthermore, disruption of the pleated septate junctions leads to defects in $D m$ BBB function (Schwabe et al., 2005; Stork et al., 2008), but the dual nature of localized xenobiotic protection mechanisms had not been established in insects.

Here, we demonstrate molecular and architectural similarities between fly and vertebrate BBBs by showing that Mdr65, a homolog of the major $\mathrm{ABC}$ transporter found at the human $\mathrm{BBB}$, 
MDR1/Pgp, is required for normal chemical protection of the $D m$ brain. We show that the fruit fly $(D m)$ is uniquely suited for live assays of BBB function, making it useful for genetic screens and real-time assessment of chemical partition phenomena. We further show that Mdr65 is specifically localized at the humoral barrier of the Dm CNS, indicating that the SPG, like the vertebrate VE, possesses both tight diffusion barriers and active efflux transporters. Our findings show strong evolutionary conservation of localized chemoprotective mechanisms and establish $\mathrm{Dm}$ as a tractable system for studying the regulatory mechanisms and integrated neuroprotective physiologies of the $\mathrm{BBB}$ in vivo.

\section{Materials and Methods}

Drosophila culture and genetics. Animals are grown on standard cornmeal molasses agar media at $25^{\circ} \mathrm{C}$ and $70 \%$ humidity in uncrowded bottles and collected $2 \mathrm{~d}$ before eclosion.

Intrahemolymph drug dosing. Dosing methods were similar to those described previously by Bainton et al. (2005). In short, intrahumoral fluor and drug doses were delivered by placing a microinjection needle between the posterior abdominal wall body segments of $\mathrm{CO}_{2}$ anesthetized animals. Positive pressure was applied to the needle under direct visualization over $1-2 \mathrm{~s}$ to deliver an average volume of $100 \mathrm{nl}$ dye per injection $\mathrm{SD} \pm 25$ (range, 70-130 nl; data not shown). Animals were allowed to recover from injection in food vials at $25^{\circ} \mathrm{C}$. In Figure 1, drugs and dyes were dosed using the following concentrations: Texas Reddextran (TRD) at $25 \mathrm{mg} / \mathrm{ml}$, FITC salt at $1 \mathrm{mg} / \mathrm{ml}$, rhodamine 123 (Rho123) at $1.25 \mathrm{mg} / \mathrm{ml}$, cyclosporin $\mathrm{A}$ (CsA) at $250 \mu \mathrm{M}$, and/or GF120918 [(N-(4-[2-(1,2,3,4-tetrahydro-6,7-dimethoxy-2 isoquinolyl)ethyl]-9,10-dihydro-5-methoxy-9-oxo-4-acridine carboxamide, hydrochloride); GF] at $50 \mu \mathrm{M}$.

Retinal images acquisition. All retinal images were taken of animals under $\mathrm{CO}_{2}$ anesthesia using methods described by Bainton et al. (2005).

In situ brain staining. Animals to be stained with C219 antibody were injected with $200 \mu \mathrm{M}$ cyclosporine A in $\mathrm{H}_{2} \mathrm{O}$ (C 1832l; Sigma) and 25 $\mathrm{mg} / \mathrm{ml} 3 \mathrm{kDa}$ Cascade blue dextran (D-7132; Invitrogen) and allowed to recover overnight. Cyclosporine A holds $\mathrm{ABC}$ B1 in an open conformation and improves C219 antibody staining in situ (Demeule et al., 1995; van Den Elsen et al., 1999). Flies are anesthetized with $\mathrm{CO}_{2}$, decapitated, and the proboscis were removed. Whole heads are placed in fixative (3.7\% paraformaldehyde in $1 \times \mathrm{PBS}$ ) for $15 \mathrm{~min}$ at room temperature. Central brains were removed from the cuticle in $1 \times \mathrm{PBS}$, carefully preserving brain surface structures, and washed with $1 \times$ PBS. Isolated brains were incubated in blocking buffer for $1 \mathrm{~h}(1 \times \mathrm{PBS}, 5 \%$ goat serum, and $4 \%$ Tween 20) and then probed in primary antibody overnight at $4^{\circ} \mathrm{C}$. Brains were washed three times for $30 \mathrm{~min}$ in $1 \times \mathrm{PBS}$ and probed with appropriate fluorescently labeled secondary antibodies for $45 \mathrm{~min}$ at room temperature (1:200; Invitrogen). Brains were washed three times for $45 \mathrm{~min}$ in $1 \times$ PBS and mounted on glass slides using DakoCytomation Fluorescent Mounting medium (Dako). Brains were mounted on glass slides with $\sim 40 \mu \mathrm{m}$ posts and then covered with coverslips and sealed.

Whole animal pharmacokinetics. At $0,1,2,4,8$, and $16 \mathrm{~h}$, hemolymphinjected whole animals were quickly frozen on dry ice and then crushed in a Microfuge tube in $250 \mu \mathrm{l}$ of $0.1 \%$ SDS and spun for $1 \mathrm{~min}$ at room temperature. Samples were diluted $10 \times$ in $0.1 \%$ SDS, vortexed, centrifuged, and $100 \mu \mathrm{l}$ placed in 96-well fluorimeter plate wells. Fluorescent units were determined using a TECAN Spectrafluor Plus fluorescence reader or Spectra-Max M2 by Molecular Devices for FITC (excitation, $485 \mathrm{~nm}$; emission, $535 \mathrm{~nm}$ ) or rhodamine B (RhoB) (excitation, $535 \mathrm{~nm}$; emission, $595 \mathrm{~nm}$ ). All samples were measured in the linear range of a standard curve for each fluor.

Brain-specific dye capture. Rhodamine B (R6626; Sigma) was dissolved in $\mathrm{H}_{2} \mathrm{O}$ at $2.5 \mathrm{mg} / \mathrm{ml}$ and brought to neutral $\mathrm{pH}$. Sibling animals of above flies were decapitated, and brains were rapidly dissected $(<90 \mathrm{~s})$ from the cuticle, trachea, and fat body in $1 \times$ PBS. With single forceps tip, brains were washed once in $1 \times$ PBS and placed in a fluorimeter plate well containing $50 \mu \mathrm{l}$ of $0.1 \%$ SDS. Brains were allowed to dissociate over 30 $\mathrm{min}$, and the dye released from brain samples was measured using appropriate filters as above. Average values of dye retained in CNS tissue were determined after subtracting the background from SDS alone. (Note that isolated brains from flour-uninjected animals contain insignificant intrinsic fluor signal.) Statistical error was calculated based on the number of brains used for each sample, because the dynamic range of the signal varies depending on the fluor used. One brain per well can be used for RhoB, and brain-specific dye capture is depicted as relative fluorescence units using SD for statistical error (see Fig. $2 B$ ). For the screen and rescue (see Figs. $2 B, 5 E$ ), RhoB data are shown as normalized values for ease of comparison across genotypes. BODIPY-prazosin (BPrz) (B-7433; Invitrogen) was dissolved in 100\% DMSO at $2 \mathrm{~mm}$ and mixed with dextrans in $\mathrm{H}_{2} \mathrm{O}$ before injection for a final concentration of $1 \mathrm{~mm} \mathrm{~B}-\mathrm{Pz}$ and $25 \mathrm{mg} / \mathrm{ml} 10 \mathrm{kDa}$ Texas Red-dextran (D-1863; Invitrogen). B-Prz provides a more limited dynamic range than RhoB; thus 10 brains were pooled per experimental replicate, and SEM was used for statistical error.

Drug efflux screen. P-element lines resident in the coding region of $\mathrm{ABC} B$ and $\mathrm{C}$ genes were collected from public sources [Bloomington Drosophila Stock Center (Indiana University, Bloomington, IN), Drosophila Genetic Resource Center (Kyoto Institute of Technology, Kyoto, Japan), and Szeged Drosophila Stock Centre (University of Szeged, Szeged, Hungary); see Flybase http://flybase.bio.indiana.edu/]. Lines tested were as follows: wild type (WT) (Canton S); a, c00522 (Mdr50); b, SZ4090 (CG1824); c, SZ EP3387 (CG4225); d, f03674 (CG3156); e, EY11060 (CG7955); f, SZ-3972 (Mdr49); g, KG08723 (Mdr65); h, EY13911 (CG5789); i, SZ430 (CG14709); j, EY09703 (CG4562); k, KG04612 (CG11898); 1, KG08719 (CG7806); m, EY11919 (CG6214); n, f05095 (CG8799); o, KG04706 (CG 31793); p, e00744 (CG5772/cyo); and q, f01338 (CG7627). Adult animals were injected with $1.25 \mathrm{mg} / \mathrm{ml}$ $\mathrm{RhoB}$ dye in water at $\mathrm{pH}$ 7.0. At $4 \mathrm{~h}$, whole animals were frozen for whole animal analysis (above), whereas sibling animal brains were dissected and CNS fluorescent dye capture was determined (as above).

Detailed pharmacokinetic analysis. All animals are injected with a mixture of $1.25 \mathrm{mg} / \mathrm{ml} \mathrm{RhoB} \mathrm{and} 25 \mathrm{mg} / \mathrm{ml} 3 \mathrm{kDa}$ FITC-dextran (D-3306; Invitrogen) (see Fig. 2D).

Cell-based efflux assay. HEK293T cells (generously provided by Dr. Warner Greene, Gladstone Institute, University of California, San Francisco, San Francisco, CA) were transfected with plasmid DNA using Lipofectamine 2000 reagent (Invitrogen) following the instructions of the manufacturer. At $24 \mathrm{~h}$ after transfection, cells were harvested and counted, and $3 \times 10^{5}$ cells were incubated in $500 \mathrm{ng} / \mathrm{ml}$ rhodamine 123 ( $\mathrm{R} 8004$; Sigma) for $30 \mathrm{~min}$ in $5 \% \mathrm{CO}_{2}$ at $37^{\circ} \mathrm{C}$. Intracellular fluorescence was measured on a FACScan flow cytometer (BD Biosciences) in channel 2 (FL2; $l_{\max }, 585 \mathrm{~nm}$ ) on a logarithmic scale. Statistical analysis was accomplished using a paired $t$ test $(p<0.05)$.

Analysis of PMdr65 insertion. Inverse PCR was performed on chromosomal DNA (Sullivan et al., 2000). PCR bands from each end of the chromosome were cloned and sequenced confirming the location for PMdr65 (KG08723) in the eighth exon of the Mdr65 gene at nucleotide 6236199 on chromosome III. Primers for reverse transcription (RT)PCR analysis were designed from the surrounding genomic sequence. Drosophila head RNA was isolated (Sullivan et al., 2000), and reverse transcription was accomplished with a unique RT primer. RT-PCR was done under standard conditions with 30 cycles, and products were run on $1 \%$ agarose gels and stained with ethidium bromide.

Gene cloning of Mdr65. Mdr65 was PCR amplified from expressed sequence tag (RE14657; Drosophila Genetic Resource Center) using pfx DNA polymerase (Invitrogen). The 5' PCR primer included an upstream CACC sequence to promote directional cloning into a pENTR/D-TOPO cloning vector (Invitrogen). Correct insertion orientation and primary nucleotide sequence (3906 bp) was confirmed by sequencing the entire open reading frame (ORF). A single amino acid change ( $G$ to $D)$ was noted at amino acid 1190. The entire ORF was moved into a upstream activating sequence (UAS) controlled Drosophila Gateway construct, pTWG, containing a green fluorescent protein (GFP) tag at the C terminus by in vitro recombination and confirmed by restriction digestion (Drosophila Gateway Cloning Collection, Carnegie Institution, Baltimore, MD). Standard transgenic methods were used to make stable UAS inducible transgenic transposon insertions into the Dm genome (Sullivan et al., 2000). 
Gene cloning of MDR1/Pgp. Human MDR1/Pgp was PCR amplified from a pcDNA-5-FRT construct furnished by the Kroetz laboratory using the same methodology as above for Mdr65. Correct insertion orientation and primary nucleotide (3840 bp) sequences for MDR1/Pgp was confirmed by sequencing the entire ORF. The MDR1/Pgp pENTR/DTOPO construct was then moved into the pTW vector for transgenic expression in Dm (Drosophila Gateway Cloning Collection). As above, standard transgenic methods were used to make stable UAS inducible transgenic transposon insertions into the Dm genome.

Western analysis. Western analysis was accomplished with standard $10 \%$ PAGE gels blotted onto polyvinylidene difluoride membranes (GE Healthcare). Primary antibody hybridization using 1:100 C219 MDR1/ Pgp Antibody (Invitrogen), 1:500 GFP monoclonal antibody (Invitrogen), or 1:50 affinity-purified Moody Beta antibody (Bainton et al., 2005) was done using a Bio-Rad Mini-gel Western protocol. Visualization of bands was accomplished by incubation with anti-rabbit alkaline phosphatase-conjugated secondary antibody and ECL solution. Mdr65GFP/SPG-GAL4 double-homozygote animals $(\mathrm{G} \times \mathrm{M} / \mathrm{G} \times \mathrm{M})$ express approximately eight times as much Mdr65-GFP as double-heterozygotes $(\mathrm{G} \times \mathrm{M})$ compared by serial dilutions into whole animal crude extracts (data not shown).

Confocal analysis. Confocal images are acquired using a Zeiss LSM-510 as described previously (Bainton et al., 2005). Laser and detector gain settings for fluorescent background noise were defined using brains with no primary antibody exposure and/or PMdr65 (an Mdr65 loss-offunction allele). At the coverslip interface, the brain was slightly pressed against the glass providing a flat brain interface with widths of $10-20 \mu \mathrm{m}$. This preparation provides highly reproducible patterns of Moody staining that allows for proper anatomic identification of dorsoventral brain orientation and overall quality of the brain preparation. Because the BBB is a continuous surface around the Dm CNS, the depth of confocality can be changed to find a cross-sectional image. To observe a tangential section, we follow the edge of the brain to its greatest extent laterally. This provides the highest resolution of the apical-basal polarization of the BBB epithelia.

Cytotoxicity assays. Vinblastine (VB) (V1377; Sigma) doses were titrated to sustain animal viability (defined by a lethal dose $<5 \%$ after overnight exposure). Control animals were injected with $3 \mathrm{kDa}$ FITCdextran. Experimental conditions included 3.3 and $6.6 \mathrm{~mm}$ vinblastine in water. Brains were dissected from live animals after overnight incubation, with two brains per well for each fluorescent measurement to increase measured signal.

\section{Results}

\section{Live assessment of chemical partition at the $D m$ retina}

To study drug transport physiology in vivo, we inject fluorescent small molecules into the hemolymph of white ${ }^{118}$ null $\left(w^{-}\right)$flies and visualize drug distribution throughout the animal by following fluorescent signal (Bainton et al., 2005). Because the Dm CNS includes the BBB-protected retinal space (Fig. $1 \mathrm{~A}$ ), xenobiotic penetration of the barrier can be observed in live flies by looking through the cornea at chemical fluors trapped in the retina (Bainton et al., 2005; Banerjee et al., 2008). The white mutation enhances the visibility of the injected dyes by eliminating dark retinal pigments that block scattered light. In previous work, we discovered Moody, a G-protein-coupled receptor (GPCR) that localizes to and regulates paracellular border function of the SPG (Daneman and Barres, 2005; Schwabe et al., 2005). Numerous highly charged fluorescent dyes or large molecular weight (MW) dextrans injected into the hemolymph compartment are excluded from the retina of $w^{-}$WT over hours and days but infiltrate $w^{-}$moody null flies within a few minutes of injection (data not shown). In the example shown in Figure $1 B$, at $4 \mathrm{~h}$ after injection, FITC salt (F) is excluded from the retina of live $w^{-}$WT animals (top, left). Note the distinct high-contrast fluorescent signal at the edge of the cornea (black triangles) demarcating the hemolymph and CNS compartments (also shown in schematic image, Fig. $1 A$, black arrow). The hemolymph exclusion line (HEL) is a consequence of differential signal intensity between hemolymph passing near the outer segments of the retina (i.e., the humoral space) and signal that originates from inside the retina (i.e., CNS space) (Fig. $1 A$, arrow). In an FITC-injected $w^{-}$ moody null animal, no HEL is observed, because FITC dye infiltrates the retina (top, right) and the central brain (data not shown). In contrast, many lipophilic dyes that are good ABC transporter substrates, such as Rho123, are excluded from the retina in both $w^{-}$WT and $w^{-}$moody null flies (Fig. $1 B$, bottom). Thus, Rho123 partitioning is not impaired by a significant defect in paracellular barrier function, suggesting that $\mathrm{Dm}$ has additional mechanisms for isolating the CNS.

Because Rho123 is an efficient substrate for the vertebrate ABC transporter MDR1/Pgp (Nag, 2003; Löscher and Potschka, 2005b), we tested whether its exclusion by the Dm BBB is affected by known MDR1/Pgp transport inhibitors. When Rho123 is coinjected into $w^{-}$WT flies with CsA or GF (Löscher and Potschka, 2005b), increased penetration of the retina by Rho123 is observed, with CsA having a stronger effect than GF (Fig. 1C, left column, middle and bottom). Neither transport inhibitor breaks down the paracellular diffusion barrier, because $10 \mathrm{kDa}$ TRD is still completely excluded from the CNS (Fig. 1C, right column). Thus, active transport appears to be necessary for maintenance of the Dm BBB and is functionally separable from the paracellular diffusion barrier.

\section{A screen for $\mathrm{BBB}$ active transporters}

The effect of CsA and GF on Rho123 partition suggested that one or more $\mathrm{ABC}$ transporters play a role in maintaining chemical isolation at the Dm humoral/CNS barrier. This assertion is supported by the exhaustive literature demonstrating that $\mathrm{ABC}$ transporters are highly expressed at many chemoprotective interfaces, including the BBB in vertebrates, and that altering their function, whether chemically or genetically, can affect xenobiotic partition (Löscher and Potschka, 2005b; Sarkadi et al., 2006). In mammals, $A B C$ genes occur in seven families (A through $G$ ), with a total of $\sim 50$ genes in humans. Interestingly, although $A B C$ gene sequence and subfamilies are highly conserved from flies to humans, the specific roles of most genes are unknown (Gerrard et al., 1993; Dean et al., 2001). The major xenobiotic transporter classes are the $\mathrm{B}(n=11), \mathrm{C}(n=12)$, and $\mathrm{G}(n=5)$ families. Unfortunately, because the best studies of $\mathrm{ABC}$ transporters in vitro and in the animal are limited to a few genes, it is not known whether they function as a complimentary system (i.e., posses chemical redundancy or compensation) or operate in chemical isolation. However, single gene loss of function can have a profound effect on chemical partition, because ABC B1 (MDR1/ Pgp), ABC C1 (MRP1), and ABC G2 (BCRP) knock-out mice demonstrate large defects in drug partition at the $\mathrm{BBB}$ when challenged with xenobiotics (Sarkadi et al., 2006). Hence, it is possible to consider experiments that phenocopy functional physiology of $\mathrm{ABC}$ transporters at the $\mathrm{Dm}$ BBB. Unfortunately, identifying such transporters purely by comparing primary sequence across phyla is not possible; thus, we focused our efforts on a reverse genetic screen of mutant alleles of $\mathrm{Dm}$ ABC genes.

Sequence comparison between human $\mathrm{ABC}$ genes and the $D m$ genome identified 22 highly homologous genes ( $\mathrm{B}, n=10$; C, $n=12$; http://blast.ncbi.nlm.nih.gov/Blast.cgi). Using fly database (http://flybase.bio.indiana.edu) searches, we identified $\mathrm{P}$-element lines that interrupted the coding region for 17 ( 7 class $\mathrm{B}$ and 10 class $\mathrm{C}$ ) of the $22 \mathrm{ABC}$ transporter genes (see Materials and Methods). All P-element lines were white plus 

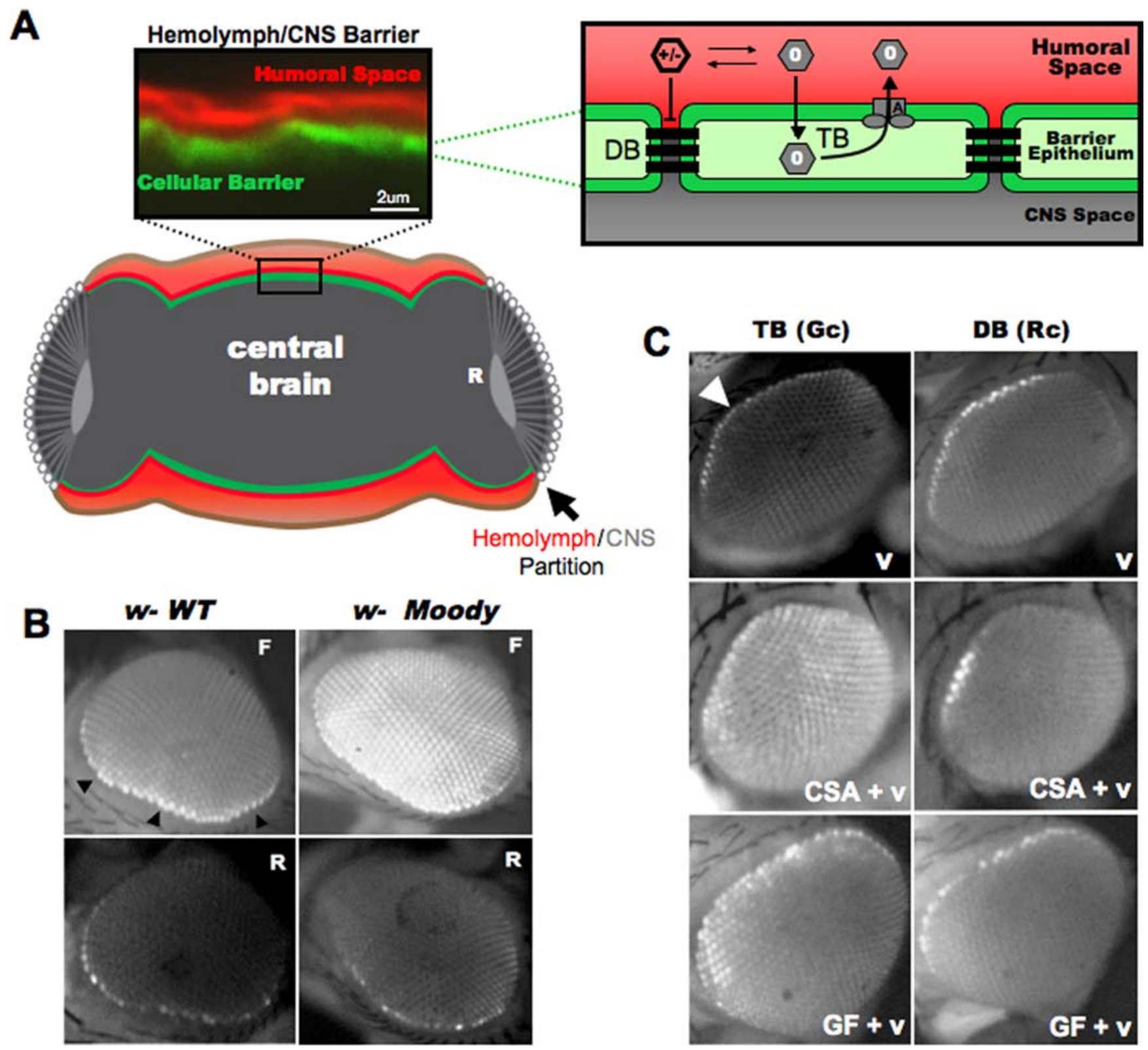

Figure 1. Visualization of drug transport in vivo through the Dm retina. A, A diagram cross section of a Dm fly head depicts different biologic spaces associated with drug partition. The CNS (gray, central brain and retina) is separated from the hemolymph (red) by an ensheathing BBB glia, the diffusion-tight SPG (green line). A confocal microscope cross-sectional image (taken in the location delineated by the black box below the image) shows the protective cellular interface (green, BBB-specific GFP) that the Drosophila brain presents to the humoral space [red, $10 \mathrm{kDa}$ dextran (for additional explanation, see Fig. 3)]. A xenobiotic (i.e., drug) interacts with protective barriers in two ways (diagram at right). Charged molecules (solid black hexagons) are excluded by the boundary function of lipid bilayers, very low rates of endocytosis, and tight diffusion barriers (DB) provided by special lateral-border junctional complexes (black boxes) of barrier epithelium. Small uncharged molecules (gray hexagons) pass easily through lipid bilayers (green), but active efflux transporters (A) move them back into the humoral space creating an active transport barrier (TB). Because drugs in aqueous solution are often in equilibrium between charged and uncharged forms, a true drug barrier must maintain both properties simultaneously to manifest xenobiotic exclusion. $\boldsymbol{B}$, Hemolymph (i.e., humoral) injection of chemical fluors allows live functional assessment of BBB function. FITC salt (F) is excluded from the retina of live $W^{-}$WT animals (top left). Black arrowheads point to the HEL, which is also shown schematically in the brain-section diagram above in $A$. FITC leaks into the retina of $w^{-}$moody null animals (top right). Rho123 (R) is excluded from the retina of $w^{-}$WT flies (bottom left) and from $w^{-}$moody null animals (bottom right). C, Simultaneous assessment of diffusion barrier and efflux transport barrier function by reporter coinjection in $w^{-}$WT at $4 \mathrm{~h}$ after injection. Rho123 demarcates the transport barrier HEL in the green channel (white triangle, TB-Gc) at top with vehicle (V), and $10 \mathrm{kDa}$ Texas Red dextran demarcates the diffusion barrier HEL in the red channel (DB-Rc). Addition of MDR1/Pgp transport inhibitors, CSA and GF, demonstrate maintenance of the diffusion barrier (right column) in the presence of a disrupted transport barrier (left column, middle and bottom).

$\left(w^{+}\right)$, precluding use of the live visual assay for small-molecule penetration screening. Therefore, dye penetration of the BBB was assessed by dissecting brain tissue from fluor-injected animals and directly measuring CNS dye capture (see Materials and Methods). The lipophilic dye Rhodamine B (RhoB) used in the screen is a substrate for $A B C$ transporters and is highly fluorescent in aqueous environments. RhoB is also well toler- ated by flies and has a bioelimination half-life of $4 \mathrm{~h}$, which is practical for quantitative assays of brain efflux (Fig. $2 A$ ). RhoB was injected into the hemolymph of 20 individuals from each $\mathrm{P}$-element line. At $4 \mathrm{~h}$ after injection, fluor content was determined for either dissected brain tissue alone or for whole animals (Fig. $2 \mathrm{~B}$ ). Whole animal fluor content showed little or no significant difference among mutant lines (black bars). 
However, one line (g) demonstrated a high relative brain-specific (colored bars) RhoB accumulation.

This line, referred to here as PMdr65, contains a P-insertion on the third chromosome in a previously described putative ABC transporter gene, Mdr65 (Wu et al., 1991; Bosch et al., 1996). The BBB phenotype of PMdr65 is retained after a fivegeneration outcross. PCR analysis of PMdr65 genomic DNA demonstrates transposon sequences resident in the eighth exon of the Mdr65 coding region, and a precise excision of the P-element restores WT drug transport (data not shown). RT-PCR analysis of PMdr65 RNA demonstrates an altered transcript consistent with $\mathrm{P}$ sequences disrupting the putative Mdr65 transporter ORF (supplemental Fig. $1 A$, available at www.jneurosci.org as supplemental material). This evidence indicates that increased brain-specific retention of RhoB results from an alteration of $M d r 65$ gene expression. $M d r 65$ exhibits $42 \%$ sequence identity to $M D R 1 / P g p$, the major $\mathrm{ABC}$ transporter found at the mammalian $\mathrm{BBB}$, and the two proteins are predicted to have similar secondary structure (data not shown).

To confirm that Mdr65 encodes an $\mathrm{ABC}$ transporter with xenobiotic efflux properties similar to MDR1/Pgp, we cloned the Mdr65 ORF by PCR, added a C-terminal GFP tag, and tested transport function of the resulting Mdr65-GFP fusion in transiently transfected HEK cells (Fig. 2C). Mock transfected cells lack sufficient endogenous ABC transporters and accumulate Rho123 when placed in dyecontaining media. In contrast, cells expressing either Mdr65-GFP or mammalian MDR1/Pgp accumulate significantly less Rho123, indicating efficient active transport of the dye out of the cytoplasm (Fig. 2C).

\section{Mdr65 loss-of-function animals are deficient in brain-specific xenobiotic efflux}

To determine whether the increased brain accumulation of RhoB in Mdr65 mutants is attributable specifically to effects on the active transport barrier, we coinjected WT and PMdr65 flies with RhoB and $3 \mathrm{kDa}$ FITC-dextran, a large MW dye conjugate that cannot be effluxed by $\mathrm{ABC}$ transporters. The total amount of each dye in whole animals or dissected brains was measured at various times after injection. As expected, RhoB is eliminated from the body within hours, whereas dextrans persist for days or weeks (Fig. 2D, and data not shown). Whole animal controls show that dosing and elimination of RhoB are not affected by the PMdr65 mutation (Fig. $2 D$, bottom), and visual inspection through the body cuticle revealed no gross differences in fluor distribution in the animal (data not shown). Furthermore, brain accumulation of 3 kDa FITCdextran remained near background levels in both WT or PMdr65 animals (Fig. $2 D$, top, green lines), indicating that the Mdr65 mutation does not significantly disturb the paracellular
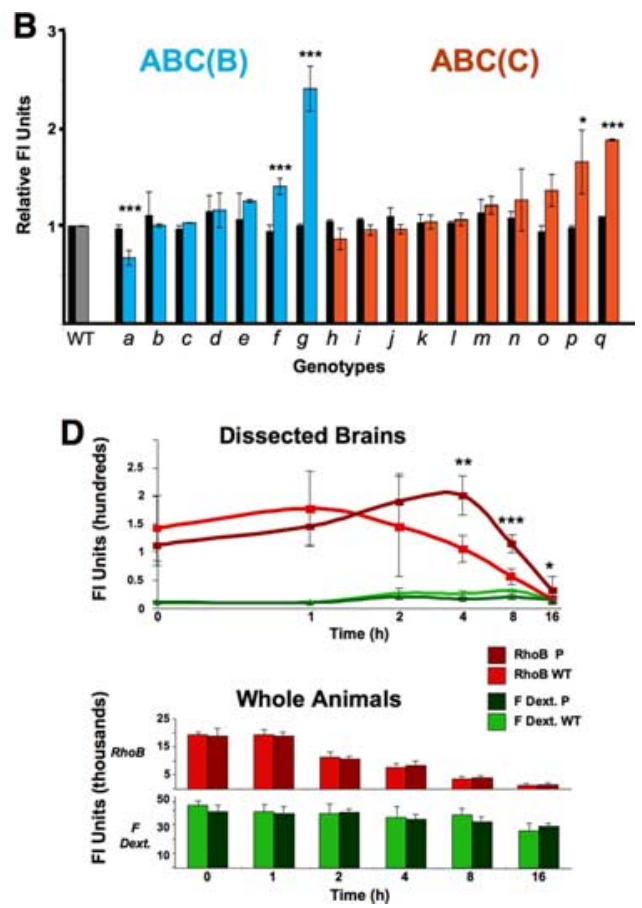

Figure 2. Mdr65 promotes efflux of xenobiotics from the brain. $A$, RhoB bioelimination from whole animals as a function of time after hemolymph injection. $\boldsymbol{B}$, Intragenic $\mathrm{P}$-element insertions in $\mathrm{ABC} B$ and $($ genes $(\boldsymbol{a}-\boldsymbol{q})$, collected from public sources, are (WT) responses in xenobiotic efflux assays (see Materials and Methods). Flies injected with $100 \mathrm{nl}$ of RhoB (black bars) and brain-specific content (colored bars). Values repre thormalized mean \pm percentage SEM for whole-body fluor content ( $n=3$ from groups of 5 crushed whole flies) or for brain (fluor content ( $n=3$ for 6 individual brains). Asterisks denote significant differences from WT control $\left({ }^{*} p<0.05,{ }^{* *} p<0.01\right.$ WT as a function of time. Animals are coinjected with $1.25 \mathrm{mg} / \mathrm{ml} \mathrm{RhoB}$ and $3 \mathrm{kDa}$ FITC - dextran (red, RhoB; green, differences in brain-specific dye capture of RhoB are seen at 4, 8, and $16 \mathrm{~h}\left({ }^{*} p<0.05,{ }^{* *} p<0.01,{ }^{* * *} p<0.001\right.$, using a animal fluor content for both RhoB (red bars) or $3 \mathrm{kDa}$ FITC - dextran (green bars) are mean \pm SD values ( $p$ values as in $\boldsymbol{a}$ ). $\boldsymbol{E}$ UAS-inducible Mdr65 RNAi [rM or VDRC ID 9019 (Dietzl et al., 2007)] is crossed to an SPG-specific GAL4 driver (G), and $G \times M$ progeny are compared with parental lines. Mean brain-specific RhoB fluor content at $4 \mathrm{~h}$ is shown \pm SD ( $p$ values same as $D$ ).

diffusion barrier. Indeed, the PMdr65 BBB was indistinguishable from WT relative to all highly charged small molecules tested (data not shown). Finally, brain levels of RhoB (red lines) were similar between fly lines at early time points, showing that the Mdr65 mutation does not affect initial brain penetration by RhoB, which was confirmed by live retinal assays (data not shown). At 4, 8, and $16 \mathrm{~h}$ after injection, however, PMdr65 flies exhibited significantly higher brain levels of RhoB than WT flies, suggesting the mutant flies had markedly reduced RhoB efflux from the brain. To confirm a role for the Mdr65 gene in BBB localized xenobiotic transport function, we produced an SPG-specific Mdr65 hypomorphic allele by crossing an SPG GAL4 driver (Fig. 3) to an inducible Mdr65specific interference RNA (RNAi) (Dietzl et al., 2007). We verified BBB-specific Mdr65 reduction by methods discussed in Figure 5 (data not shown). Like PMdr65 individuals, these animals are deficient in brain-specific RhoB transport when compared with WT (Fig. 2E). These findings parallel the brain-specific pharmacokinetics of MDR1/Pgp substrates in $M d r 1 a-M d r 1 b$ knock-out mice, demonstrating that the contribution of Mdr65 to BBB drug efflux function in Dm is similar to that of MDR1/Pgp in vertebrates. (Schinkel, 1997; Schinkel et al., 1997). 

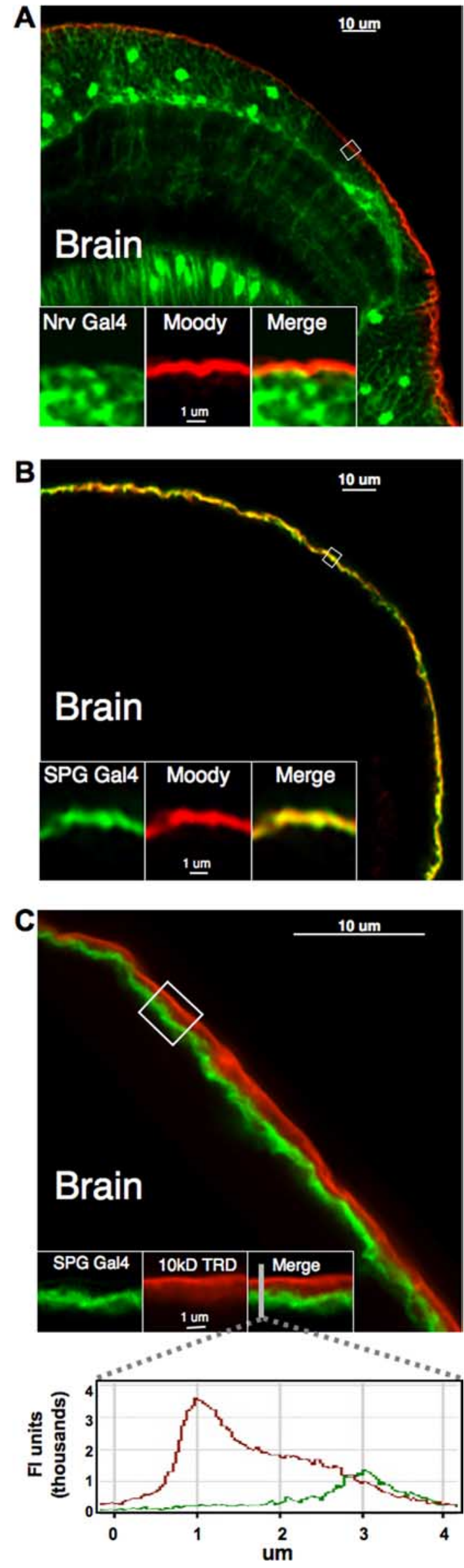

Evaluating barrier physiology at the subperineural glia in adult Drosophila

Previously, the subperineural glia was identified as the potent CNS diffusion barrier layer in EM studies in other insects and in Drosophila larva (Treherne, 1972; Carlson et al., 2000; Stork et al., 2008). In recent work, we showed that a novel orphan GPCR, Moody, is expressed exclusively in the SPG layer of larva brains and nerves (Bainton et al., 2005). To correlate in vivo assays at the retina (Fig. 1) with functional changes at the barrier, we developed methods to simultaneously identify the anatomic localization and physiologic properties of the humoral/CNS interface in $D m$ adults. Fixation is performed in situ to preserve BBB anatomy susceptible to damage during dissection of the exoskeleton (see Materials and Methods). To accomplish this, the proboscis is removed to allow access of fixative into the brain. Although the $\mathrm{BBB}$ anatomy around parts of the central brain is distorted by this procedure, subsequent dissections and mountings enable reproducible visualization of the BBB in the area of the lobular plate, the optic chiasm, and retina (Fig. 1). The retina is often removed during mounting for better position of the brain on the slide. By costaining brains expressing Nrv2-GAL4, a gene whose expression is limited to neurons and cortical glia (Pereanu et al., 2005), we show that Moody antibody staining in adults is peripheral to the CNS (Fig. 3A). Higher-resolution images confirm that the Moody GPCR is superficial to cortical glia, discrete to the SPG, and thus specifically demarcates the Dm BBB border in situ (Fig. $3 A$, inset).

A hallmark of BBB physiology is the compact cellular localization of chemoprotective systems that include tight junctional barriers and $\mathrm{ABC}$ transporters (Fig. 1A). To understand how specific gene expression at the $\mathrm{BBB}$ interface generates cellautonomous physiologic function, we used methods to control gene expression in the SPG while concurrently evaluating physiologic outcomes at that interface. Targeted gene expression is achieved using the two transgene method (Brand and Perrimon, 1993). The transcriptional activator GAL4 was put under the control of the Moody transcriptional enhancer to produce a highly specific GAL4 expression pattern specific to the subperineural glia layer (SPG-GAL4) (Stork et al., 2008). Moody has two isoforms, $\alpha$ and $\beta$, that completely colocalize (Bainton et al., 2005). SPG-GAL4-specific gene expression of a GFP-tagged Moody $\alpha$ construct coincides perfectly with Moody $\beta$ antibody staining (Fig. $3 B$, inset), verifying that gene expression can be directed exclusively to the SPG cell layer in adult flies. This expression specificity confirms that Mdr65 RNAi-induced loss of function in the SPG is targeting a BBB localized chemoprotective function of Mdr65 (Fig. 2E).

\footnotetext{
Figure 3. Confocal identification of the Dm humoral/CNS interface. $A$, Moody localizes to the SPG in adult brains with Moody $\beta$ antibody staining above Nrv2-GAL4 in a single highresolution confocal slice tangential to the CNS-hemolymph interface. High-magnification images of Nrv2-GAL4 $\times$ UAS-GFP (inset left) and Moody antibody (inset center) show no colocalization of signal (inset right). $B$, SPG-GAL4 expression colocalizes with Moody. SPG-GAL4 crossed to UAS-Moody- $\alpha$-GFP (green, SPG-GAL4) demonstrates discrete expression in the SPG of an adult brain (inset left). Costaining with Moody $\beta$ antibody (red, Moody) demonstrates native Moody localization discretely in the $B B B$ (inset center). The merged image demonstrates colocalization of both signals, showing that the SPG-GAL4 recapitulates the Moody expression pattern (inset, right). C, The SPG-GAL4 identifies the strong diffusion barrier in situ. Lysine fixable $3 \mathrm{kDa}$ TRD is injected into the fly hemolymph and allowed to mix in the circulation and fixed in situ. A high-magnification cross-sectional confocal image of brain removed from the fly cuticle shows the SPG layer (inset left) inside the bright dextran signal (inset center) fixed to brain surface tissue. No signal passes the SPG layer (merge and histogram); thus, the tight diffusion barrier-humoral interface is demarcated by the SPG-GAL4.
} 
A

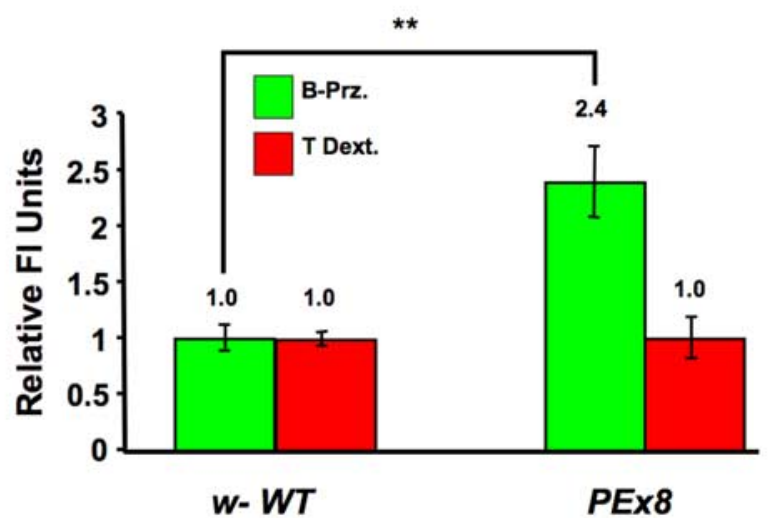

C

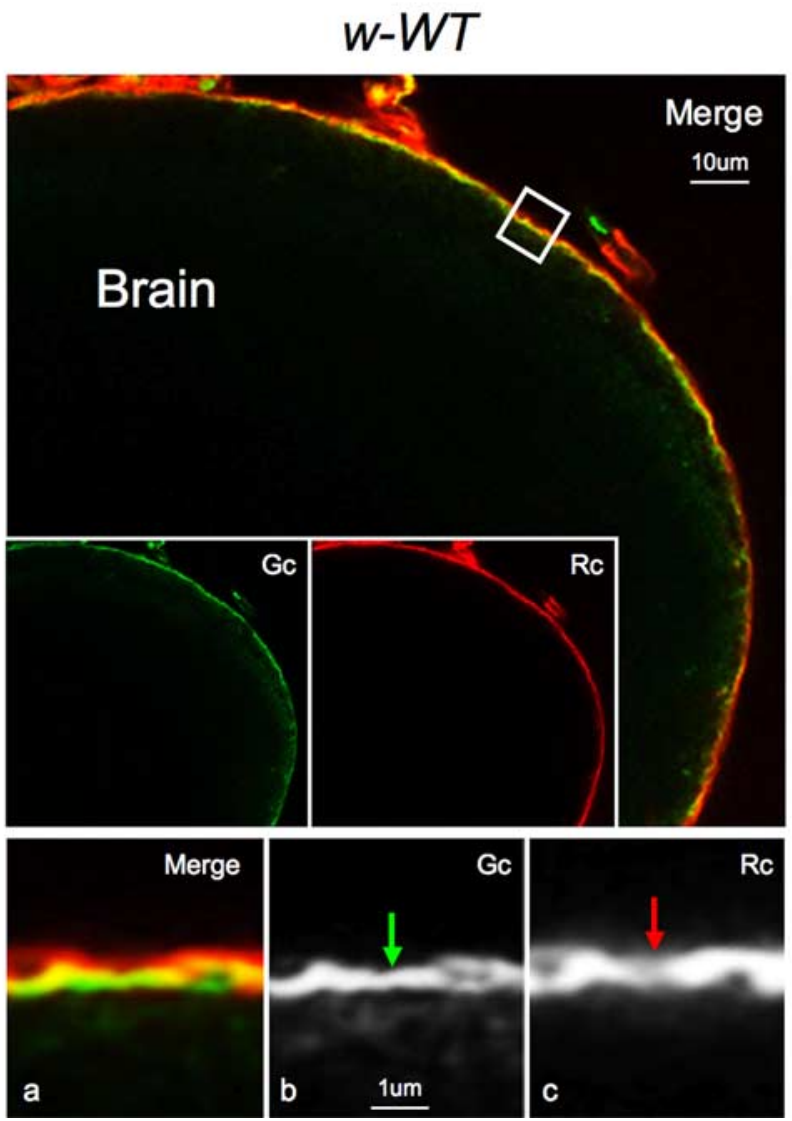

B

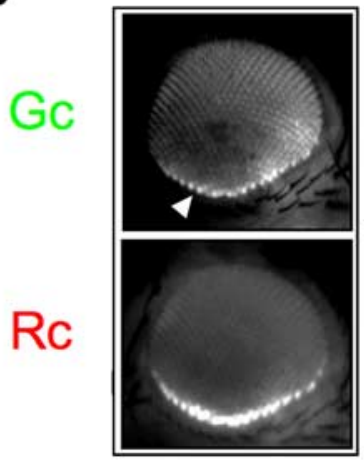

w- WT

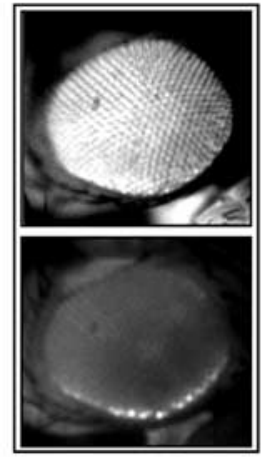

PEx8

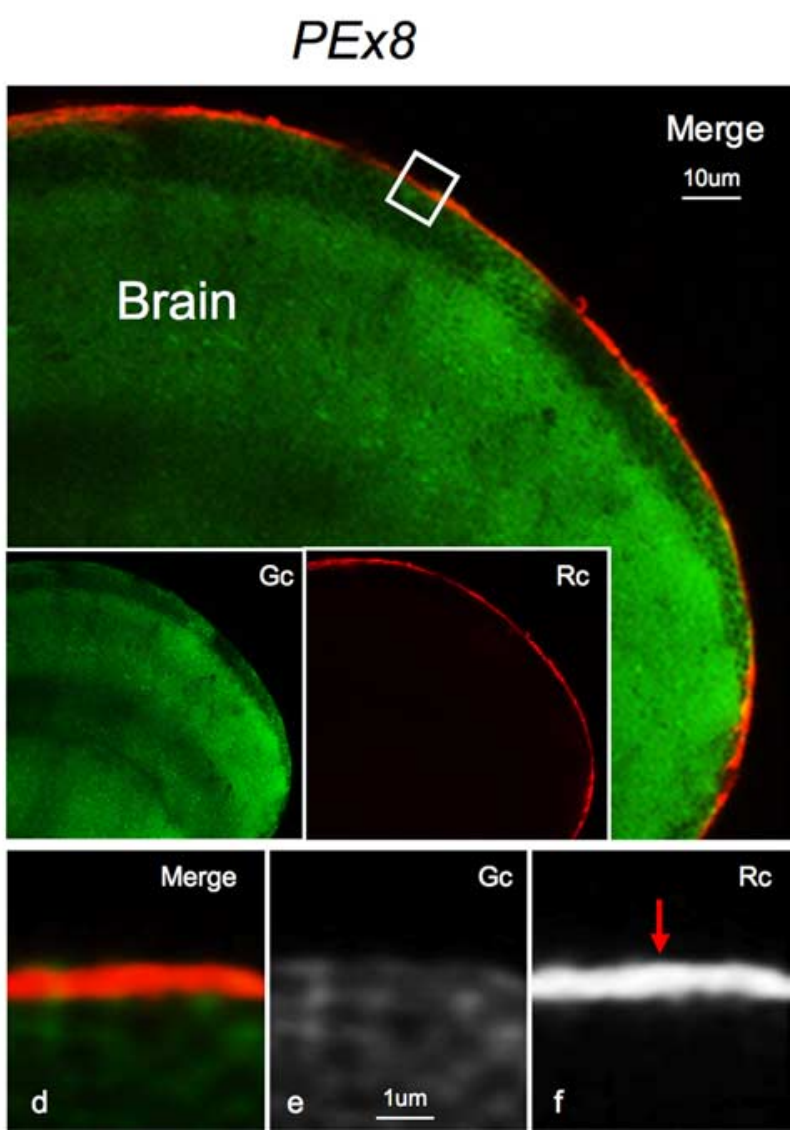

Figure 4. Mdr65 promotes drug exclusion at the SPG. A, WT and Ex8 are coinjected with $10 \mathrm{kDa}$ Texas Red dextran (red bars) and $1 \mathrm{~mm}$ B-Prz (green bars). The solid bracket line compares normalized mean \pm SEM values ( $n=2$ with 10 brains per value) of $w^{-}$WT and PEx8 ( $w-$ mdr65 null) brain-specific fluor content (4h after injection). $\boldsymbol{B}$, Live retinal images of B-Prz distribution [green channel (Gc)] show marked changes in small-molecule distribution in PEx8 (loss of HEL) compared with $w^{-}$WT (with HEL, triangle), whereas high MW dextrans [red channel (Rc)] retain normal HELs in both lines. C, Precise anatomic position of the CNS- humoral interface is shown by confocal cross-sectional images of the posterior medulla in fixed and dissected brains. In $W^{-}$WT brains, the intact diffusion barrier, demarcated by the extent of penetration of $10 \mathrm{kDa}$ TRD (red), is seen as a red line at the edge of the brain parenchyma (merge and Rc). B-Prz infiltrates the brain of PEx8 animals (compare top, $w^{-}$WT and PEx8). By separating the fluorescence channels a second high-contrast line in the Gc emerges in $w^{-}$WT only. (White boxes demark the localization of the humoral interface seen at higher magnification in bottom panels.) $\boldsymbol{b}$ identifies a tightly localized B-Prz line identifying a small-molecule transport barrier (green arrow) near the diffusion barrier seen in c (red arrow). On right, a complimentary image shows an PEx8 brain lacking the transport barrier fluorescent signal (e) but normal diffusion barrier panel ( $\boldsymbol{f}$ ) (red arrow).

Localized physiologic properties of the BBB are evaluated by combining fluorescent chemical reporters and the above anatomic methods. Standard confocal samplings methods yield a detailed cross-sectional image of the humoral interface, demonstrating that the barrier is closed to $10 \mathrm{kDa}$ dextrans along the entire periphery of the brain (Fig. $3 C$ ). Indeed, these barrier properties correlate well with dextran diffusion experiments at the vascular endothelium of the vertebrate BBB (Ballabh et al.,
2004). Note that some dye is trapped by a more superficial cellular layer known as the perineural glia (PG) (Stork et al., 2008), resulting in a gradient along the brain surface (Fig. $3 C$, inset). However, whereas the PG is completely infiltrated with dye, no fluorescent signal transits the SPG cellular barrier layer (Fig. 3C, histogram), identifying the SPG as the tightest diffusion barrier at the humoral interface. In situ localization of dextran penetration correlates well with live retinal assays of 

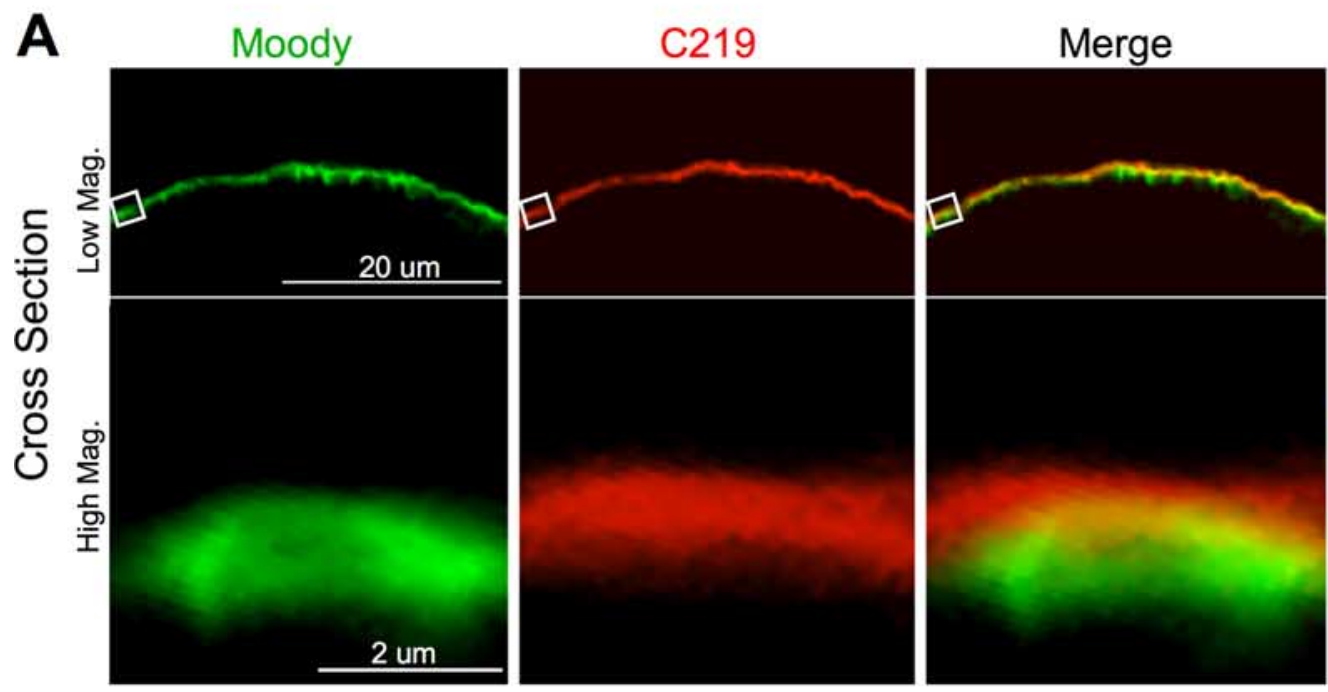

Merge

+ Diffusion Barrier
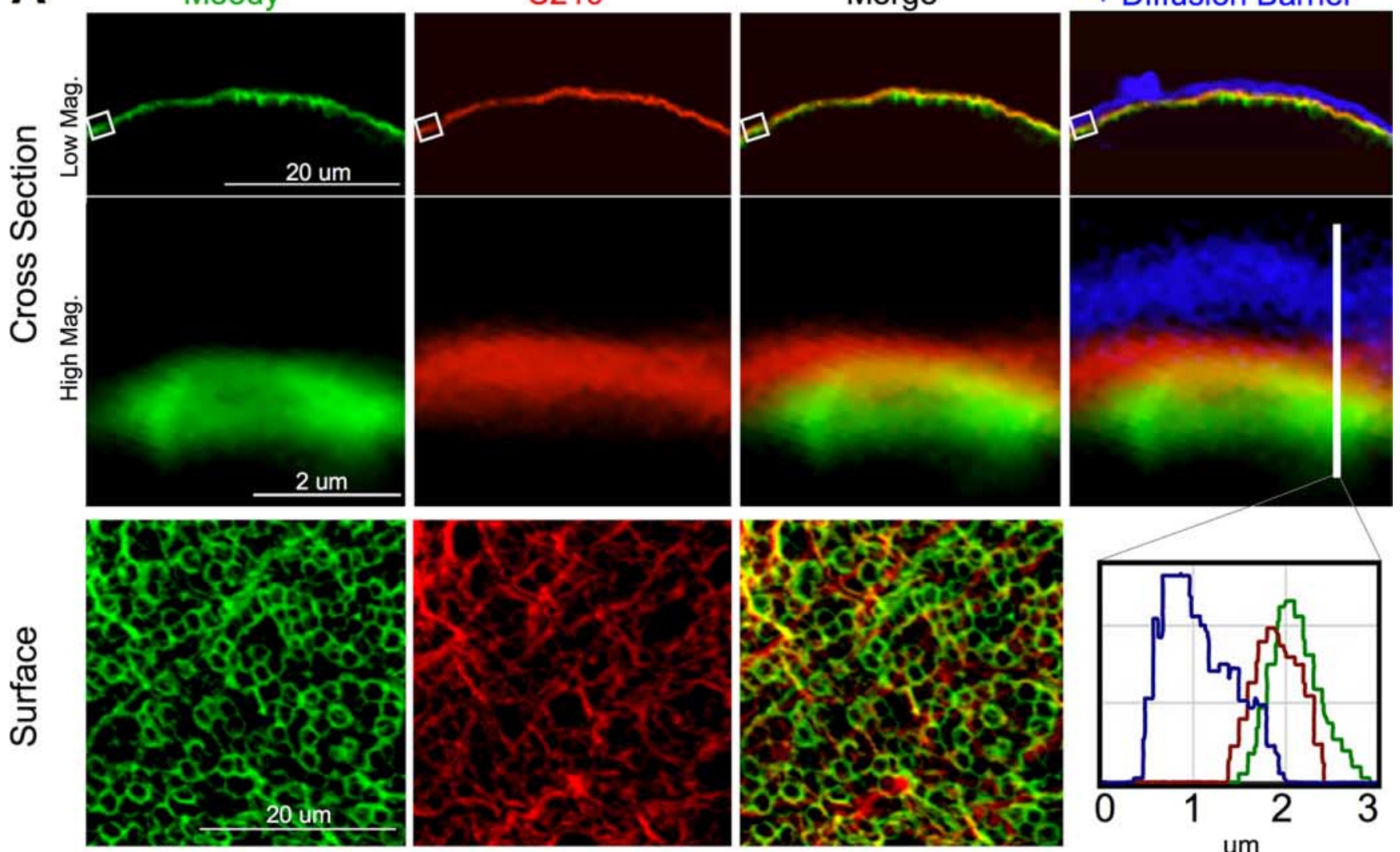

B

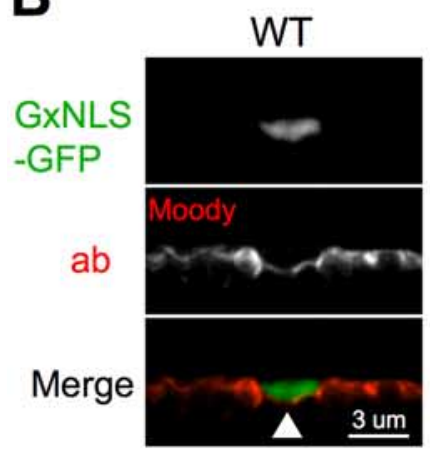

D

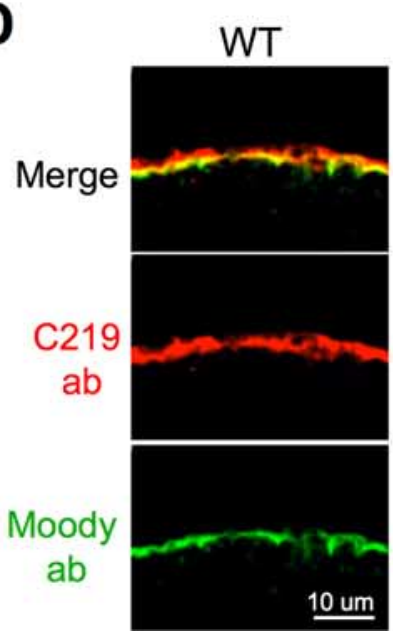

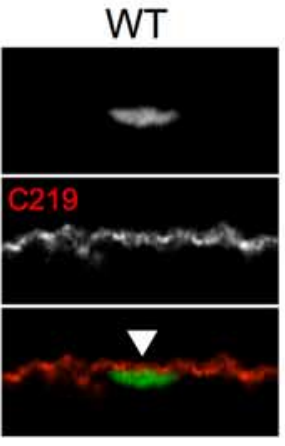

PMdr65

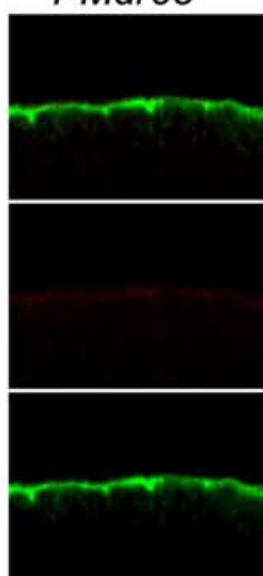

C

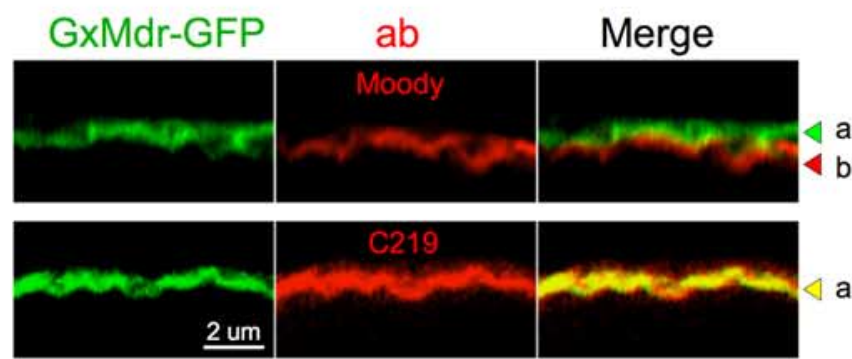

E

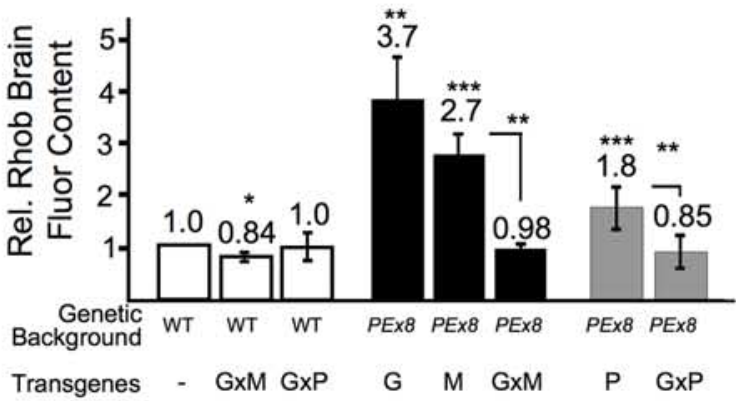

F

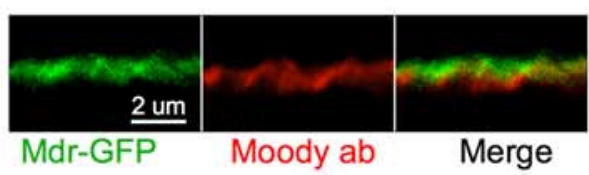


BBB function (Fig. 1) and fluor content of brains in quantitative dissection assays (Fig. $2 D$ ). Together, these data show that the $D m$ SPG in adults is a CNS ensheathing epithelium distinct from other glial layers with tight-diffusion barrier properties similar to the vascular endothelium.

It is important to note that the vertebrate $\mathrm{BBB}$ is complex, comprising multiple cell types (i.e., capillary vascular endothelium, pericytes, a basement membrane, and closely associated astrocytic glia) (Abbott, 2005). Furthermore, it participates in numerous cellular physiologies, including hemodynamic neurovascular coupling, neuroimmune function, extracellular matrix interactions, neurotransmitter inactivation, and, of course, chemical protection (Zlokovic, 2008). The integration of these physiologies requires a dynamic barrier, because it must respond to changes in metabolic requirements as well as to exogenous threats, such as xenobiotics and infectious agents. Similar to vertebrates, the $\mathrm{Dm}$ BBB has multiple cell types at the $\mathrm{CNS} /$ humoral interface. Moving outward from neurons the $D m$ barrier includes two glially derived cell layers (subperineural and perineural) (Fig. $3 C$ ), an associated immune cell layer and a fat body layer (data not shown). The primary focus of this work is the physiologic similarity of the vascular endothelium in vertebrates and the subperineural glia in $\mathrm{Dm}$. However, the additional cellular complexity of the Dm BBB may offer ways to model cellular interactions in regulation and control of $\mathrm{BBB}$ physiology, including sensing and responding to the metabolic needs of neurons, immune cell transcytosis, and remodeling after CNS injury. Thus, although the Dm BBB is not anatomically superimposable on that of vertebrates, this work establishes a framework on which to build an integrated model of BBB physiology and to discover and test regulatory hypotheses of CNS chemical protection.

\section{Mdr65 drug partition function localizes to the CNS/humoral interface}

To better understand chemoprotective deficits of Mdr65 loss-offunction animals, we compared the chemical partition of a num-

\footnotetext{
$\leftarrow$

Figure 5. Mdr65 localizes specifically to the SPG. $\boldsymbol{A}$, Confocal microscope cross-sectional images show tangential slices of the hemolymph-brain interface (top panels) taken from animals injected with $3 \mathrm{kDa}$ Cascade blue dextran (CBD) and cyclosporine A (see Materials and Methods). Brains are fixed in situ, dissected, and costained with Moody (green) and C219 (red) antibodies. The top panel shows Moody and ( 219 signal tightly and evenly colocalized along the brain surface (Merge). This signal is juxtaposed against the humoral interface [i.e., the diffusion barrier (DB)] marked by CBD (blue in Merge + Diffusion Barrier). C219 signal (red) is localized predominantly between the humoral interface (blue) and Moody (green) (high magnification, middle panel and histogram). A surface image (bottom panels) demonstrates different localization patterns of Moody and C219 signal in the plane of the SPG. B, SPG - GAL4 driver (G) (Stork et al., 2008) is crossed to UAS-GFP tagged with a nuclear localization signal [NLS-GFP (Bloomington Drosophila Stock (enter)]. Brains are stained with Moody or C219 antibody. Crosssectional images of the humoral interface show Moody stain is basal to SPG nuclei (triangle, left panel), and (219 stain is immediately apical (triangle, right panel). C, UAS-Mdr65-GFP (M) is crossed to the SPG-GAL4 driver (G) and stained with Moody antibody (top) or C219 (bottom). Mdr65-GFP localizes in the humoral facing membrane of the SPG $(\boldsymbol{a}$, green arrowhead) apical to Moody ( $\boldsymbol{b}$, red arrowhead). Mdr65-GFP and C219 stain completely colocalize (merge, yellow signal) in the apical SPG (bottom panel, $\boldsymbol{a}$, yellow arrowhead). $\boldsymbol{D}$, WT and PMdr65 animals are costained with Moody (green) and C219 (red) antibodies. Moody intensity is the same in both lines (bottom panel). Under the identical confocal conditions, C219 staining appears markedly reduced in the PMdr65 line (right middle panel). $\boldsymbol{E}$, Top, The transgenes SPG-GAL4 (G), UASMdr65-GFP (M), and UAS-Pgp (P) are crossed alone or together $(G \times M$ or $G \times P)$ into $P E x 8$ or $w-$ WT flies. Brain-specific dye capture at $4 \mathrm{~h}$ after injection of $2.5 \mathrm{mg} / \mathrm{ml} \mathrm{RhoB} \mathrm{is} \mathrm{shown} \mathrm{as}$ mean \pm SEM values $\left(n=4-8\right.$ with 3 brains per $n ;{ }^{*} p<0.5,{ }^{* *} p<0.01,{ }^{* * *} p<0.001$, for two-tailed $t$ test) (WT vs $P E \times 8 / G \times M, p>0.80$; WT vs PEx8/G $\times P, p>0.81$ ). $F$, Confocal image of $P E x 8 / G \times M$ animal brains stained with Moody antibody (red). Mdr65-GFP (green) demonstrates the correct apical localization in the SPG.
}

ber of fluorescently tagged MDR1/Pgp substrates in $w^{-}$WT flies and $w^{-}$PEx8 flies using the live retinal assay. PEx8 is a loss-offunction allele of $M d r 65$ derived from an imprecise excision of PMdr65 (supplemental Fig. S1 A, available at www.jneurosci.org as supplemental material). We found that B-Prz partition was affected by loss of Mdr65 function (data not shown). Coinjection of TRD and B-Prz into $w^{-}$WT flies and $w^{-}$PEx8 flies confirmed that the loss of Mdr65 function in PEx8 flies leads to increased levels of B-Prz in both the brain and retina but leaves the paracellular diffusion barrier intact (Fig. 4A,B). These data are directly visualized in CNS tissue by confocal microscope sections of whole dissected Dm brains. Brains from hemolymph-injected adult animals demonstrate clear penetration of B-Prz throughout the CNS parenchyma in PEx8 animals but tight exclusion from the brains of WT animals (Fig. 4C). A striking feature of these images is the tight juxtaposition of B-Prz signal (green) near the diffusion barrier (red) in WT flies, suggesting that the efflux transport barrier, like the diffusion barrier, is closely localized at the humoral/CNS interface (Fig. $4 C, w^{-}$WT, top panels and small panel $b$ ). In contrast, the transport barrier is absent in PEx8 flies (Fig. $4 C$, compare small panels $b-e$ ). Thus, brain-specific Mdr65 drug partition function localizes to the humoral interface. Furthermore, the close proximity of the diffusion and transport barriers in the SPG recapitulates the simultaneous manifestation of the diffusion and transport barriers in the vertebrate vascular endothelium (Fig. 1).

\section{Mdr65 is specifically localized in the Dm CNS}

The distinctive BBB exclusion pattern of B-Prz suggested that Mdr65 transport function is located in the SPG. Indeed, Mdr65 mRNA is known to be highly expressed in the brain surface glia of $D m$ and regulated by glial-specific transcription factors (Freeman et al., 2003). To ascertain the precise location of endogenous Mdr65, we combined newly identified Mdr65-specific antibody reagents with the above methods for understanding BBB structure and physiology. Thus, we bring together three types of reagents: antibodies that specifically recognize $\mathrm{ABC}$ transporters in the cell membrane, enhancer traps specific to the different cellular layers of the $\mathrm{Dm} \mathrm{BBB}$, and fluorescent dextrans that demonstrate the diffusion constraints of the cellular junctions of the $\mathrm{BBB}$. Together, these tools allowed us to specifically visualize individual cellular layers of the very compact humoral/CNS interface.

To this end, we confirmed that C219, an MDR1/Pgp-directed monoclonal antibody, specifically recognizes Mdr65-GFP by Western analysis of whole fly heads (supplemental Fig. S1C, available at www.jneurosci.org as supplemental material) (Kartner et al., 1985; Riordan et al., 1985; Bosch et al., 1996). Crosssectional images of adult brains costained with Moody and C219 antibodies demonstrate continuous colocalization of Moody and Mdr65 at the periphery of the CNS (Fig. 5A, top row). Higherresolution images obtained by repeated confocal sampling show that Mdr65 is predominantly apical to Moody all along the SPG/ humoral interface (Fig. 5A, middle row and inset histogram). Brain-surface images reveal different patterns for Moody and Mdr65 in the $x-y$ dimension, suggesting different restricted localizations at the humoral interface (Fig. $5 \mathrm{~A}$, bottom). We further refined relative localization of the two signals by labeling SPG nuclei with GFP. Because SPG nuclei are 500-800 nm in thickness, the distance between the apical and basal membranes of the SPG is increased near the nucleus (Fig. 5B). Under such conditions Moody is clearly localized to the basal side of nuclei, whereas Mdr65 resides on the apical side. To determine whether 
Mdr65 and Moody are localized to the same cell, we stained animals expressing low levels of SPG-specific Mdr65-GFP with Moody antibodies and found that Mdr65-GFP is apical to Moody with little or no overlap (Fig. 5C, top). When the same animals are stained with C219, the GFP and C219 signals are perfectly coincident, indicating that Mdr65 colocalizes with Mdr65-GFP and therefore resides in the apical interface of the SPG (Fig. 5C, bottom). Finally, C219 staining of WT and PMdr65 brains demonstrates greatly reduced signal at all localizations along the BBB interface in PMdr65 animals, confirming that PMdr65 is a loss-of-function allele of $m d r 65$ (Fig. 5D, middle).

\section{SPG-specific expression of Mdr65-GFP or human Pgp rescues transport function}

To confirm that Mdr65 loss of function is responsible for functional perturbations in BBB-specific xenobiotic efflux, we showed that expression of Mdr65-GFP in the SPG rescues the RhoB transport phenotype. Targeted gene expression was achieved using an SPG-GAL4 transgene $(\mathrm{G})$ driving the expression of UAS-Mdr65-GFP (M). Brain-specific efflux of RhoB in a WT background is only modestly affected by a single $\mathrm{G} \times \mathrm{M}$ transgene cross (Fig. 5E, column 2). However, the same cross completely restores WT transport function in an Mdr65 null (PEx8) background (Fig. 5E, column 6). Apical SPG localization of Mdr65-GFP was confirmed in rescued animals by confocal microscopy (Fig. 5F). Thus, Mdr65-GFP expression at the humoral interface is sufficient to restore efflux transport of smallmolecule fluors, indicating that a role of Mdr65 in CNS protection is localized to the SPG. These data suggest that Mdr65 plays a cell-autonomous role in xenobiotic protection at the fly BBB similar to the proposed role of other powerful $\mathrm{ABC}$ transporters at the VE of vertebrates (Sarkadi et al., 2006). If so, then Mdr65 loss of function should be complemented by a similarly functioning vertebrate gene. We tested this hypothesis by driving the expression of human MDR1/Pgp in the Dm SPG (G) with a UASMDR1/Pgp construct (P) and confirmed protein expression in Western blots probed with C219 antibody (supplemental Fig. S1D, available at www.jneurosci.org as supplemental material). $\mathrm{G} \times \mathrm{P}$ animals expressing low levels of MDR1/Pgp in a WT background had no effect on RhoB brain retention (Fig. $5 E$, column $3)$. Animals carrying, but not selectively expressing, the P transgene in an Mdr65 null (PEx8) background could partially rescue the mutant phenotype, likely secondary to leakiness of the transgene construct (Fig. 5E, column 7). However, a $\mathrm{G} \times \mathrm{P}$ transgene cross in the mutant background fully restored WT levels RhoB transport (Fig. 5E, column 8). Thus, evolutionarily distant, but physiologically similar, xenobiotic transporters can promote similar levels of chemical protection function across species.

\section{SPG Mdr65 levels alter BBB susceptibility to cytotoxins}

Drug transport studies in vivo and in vitro suggest that ABC transporters are modular units and that efflux function is proportional to transporter expression levels (Dohgu et al., 2004; Löscher and Potschka, 2005a; Bachmeier et al., 2006; Sarkadi et al., 2006). To determine whether similar properties would manifest in vivo at the humoral/CNS interface of Dm, we tested the effect of Mdr65GFP overexpression on RhoB partitioning. Transporter gain-offunction conditions were achieved using double homozygotes of the $\mathrm{G}$ and $\mathrm{M}$ transgenes $(\mathrm{G} \times \mathrm{M} / \mathrm{G} \times \mathrm{M})$ in a WT background. Mdr65-GFP expression is several fold greater than native Mdr65 expression in WT animals (supplemental Fig. S1C, available at www.jneurosci.org as supplemental material), and all Mdr65GFP transporter localization is at the apical interface of the SPG (data not shown). Brain-specific RhoB content is significantly decreased in $\mathrm{G} \times \mathrm{M} / \mathrm{G} \times \mathrm{M}$ lines relative to $\mathrm{WT}$ (Fig. $6 B$ ). Thus, increasing or decreasing the level of ABC transporters in the $\mathrm{Dm}$ SPG can alter xenobiotic partition at the humoral/CNS interface in much the same way that changes in MDR1/Pgp expression alters xenobiotic penetration into the cytoplasm of isolated cultured cells (Chaudhary and Roninson, 1993).

Pharmacokinetic models predict that altering the level of $\mathrm{ABC}$ transporter expression or localization will shift the chemical partition of drugs in the BBB-protected space (Schinkel, 1999). Because Mdr65 resides in the apical membrane of the SPG in flies, the cytoplasm of SPG cells is included in the BBB-protected zone, and varying the level of Mdr65 should alter the sensitivity of SPG cells to ABC transportable cytotoxins in a predictable manner. To test this hypothesis, we studied the effect of the anti-microtubule agent vinblastine on SPG cell function. VB was chosen because it is transported by Mdr65 in vitro (data not shown). Because breakdown of the cytoskeleton affects the integrity of paracellular junctions, SPG cell function can be assayed by monitoring the passage of large MW dextrans from the hemolymph into the CNS. In the absence of $\mathrm{VB}$, the paracellular diffusion barrier is intact, and the $3 \mathrm{kDa}$ FITC-dextran is entirely peripheral to the brain (Fig. $6 \mathrm{C}$ or $D$, and data not shown). When WT animals are coinjected with $3.3 \mathrm{~mm}$ VB and FITC-dextran, a small but significant amount of FITC signal leaks into the CNS (Fig. 6C, left). Under the same conditions, Ex8 brains accumulate FITC-dextran internal to the SPG more than WT brains, indicating that $m d r 65$ loss of function increases the sensitivity of SPG cells to VB (Fig. 6 C, right, and data not shown). Similar results are obtained when Mdr65 loss of function is directed to the SPG by Mdr65 RNAi (Fig. 2E); thus, cell-autonomous Mdr65 function provides some measure of chemoprotection at the barrier interface (data not shown). We performed the same experiment on $m d r 65$ overexpressors using 6.6 $\mathrm{mm}$ VB. These animals accumulated significantly lower levels of FITC-dextran than WT (Fig. $6 D, \mathrm{G} \times \mathrm{M} / \mathrm{G} \times \mathrm{M}$ ). In vivo retinal dye penetration studies corroborated the results obtained above by direct assessment of retinal fluor content (Fig. 6C,D, bottom panels). These results indicate that anatomically selective changes in Mdr65 can lead to altered levels of chemical neuroprotection and suggest that modulation of individual components of the $\mathrm{BBB}$ can be exploited to adjust the entry of specific drugs into the brain.

\section{Discussion}

The CNS is protected from the influence of the external environment by a blood-brain barrier. This cellular layer uses two properties to promote neuroprotection: a tight diffusion barrier and a complex array of transcellular transporters. Although both properties are essential for proper humoral/CNS separation, little is known about their functional integration and regulation. In the Drosophila BBB tissue layer, the SPG, strong diffusion barrier properties had been identified previously, but the nature of its xenobiotic barrier had not been established. In this study, we characterize both physiologic aspects of the adult animal barrier and describe a novel system for the study of brain-specific smallmolecule transport physiology. We combine in vivo physiologic assays for drug barrier function and forward genetics to identify Mdr65 as an essential BBB transporter. Mdr65 loss of function leads to increased accumulation of $\mathrm{ABC}$ transporter substrates in the brain and increased sensitivity to cytotoxic xenobiotics. These studies suggest functional parallels between Mdr65 and the human MDR1/Pgp transporter and together show strong evolutionary conservation of cell structure 
and chemoprotective mechanism in vertebrate and invertebrate CNS/humoral interfaces.

Chemical protection of the brain is a complex process involving many overlapping physiologic systems and thousands of genes (Abbott, 2005; Sarkadi et al., 2006; Zlokovic, 2008). Although recent advances in genomic and proteomic profiling of $\mathrm{BBB}$ components promise to provide a detailed description of the molecular players in BBB physiology, understanding how these components act in concert in a given environment remains a difficult problem for vertebrate systems to solve (Calabria and Shusta, 2006). Integrative physiology is a discipline that promotes the use of appropriate model organisms to test physiologic function of particular genes and interacting genetic systems (Dow, 2007). A powerful ambition of this approach is to find an experimental system advantageous for interpretation of gene function in different functional contexts (i.e., in the whole animal and/or cell-autonomous/tissue-based circumstances) (Yang et al., 2007). We chose to focus our work in Drosophila in which a glial-dependent blood-brain barrier chemically insulates an open circulatory system from the retina, central brain, and peripheral nerves (Carlson et al., 2000; Stork et al., 2008).

Previously, we discovered and began to characterize the BBB-specific function of a Dm orphan GPCR, Moody, that localizes with junctional complex components and likely controls diffusion barrier tightness through signals to the actin cytoskeleton (Bainton et al., 2005; Schwabe et al., 2005). The functional association of Moody with cellular junctions demonstrated for the first time the existence of hierarchical control systems designed to direct specific aspects of $\mathrm{BBB}$ physiology. Interestingly, varying degrees of hypomorphic mutants in Moody demonstrate a range of phenotypes from subtle behavioral changes (i.e., drug responses) to outright disruption of the diffusion barrier in null animals. Because GPCRs transmit information from external cellular stimuli as varied as photons and hormones, the discovery of Moody suggested the possibility that chemoprotective sensors may localize to and provide critical moment to moment evaluation and adjustment of barrier performance. However, to pursue the control systems of chemical protection physiology, additional molecular and cellular components of the Dm CNS xenoprotective interface had to be established (Fig. 1).

ABC transporters control localized pharmacokinetic penetration of drugs and are highly homologous between species; thus, it was logical to pursue their role at the Dm BBB. Unfortunately, direct sequence comparisons of human MDR1/Pgp or Mrp1 to Dm ABC B and C gene family members, respectively, did not yield any obvious candidates for specific chemoprotective genes
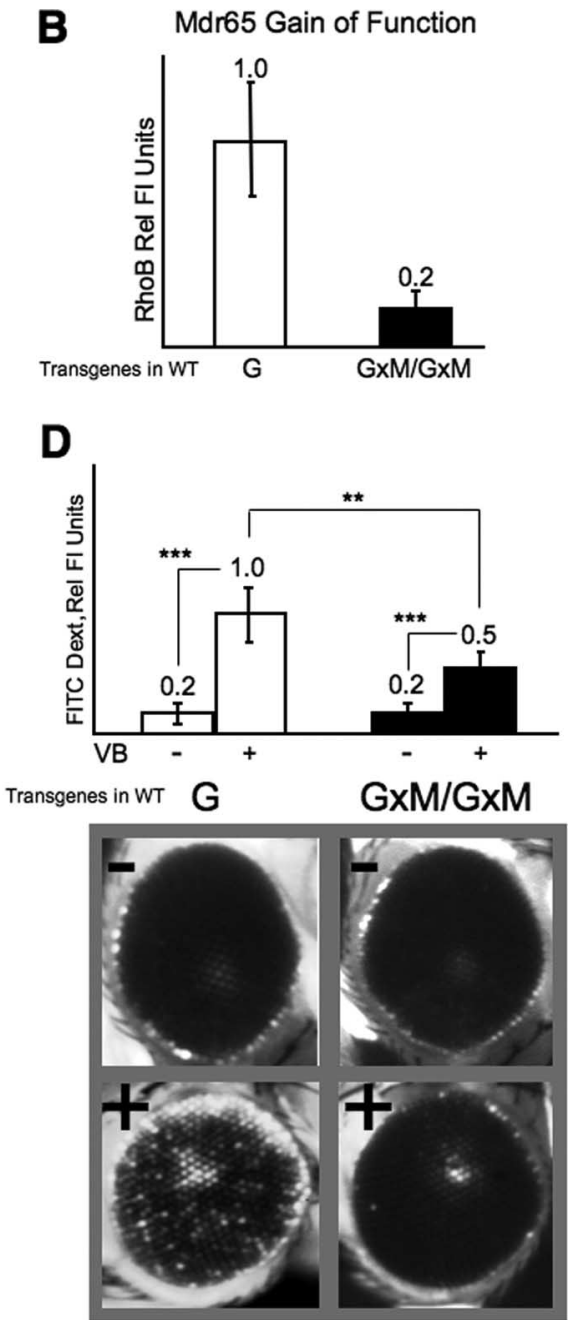

Figure 6. Mdr65 levels change the sensitivity of the Dm BBB to cytotoxic drugs. $A$, Relative brain flour content of $P E x 8$ animals is compared with $w-$ WT at $1.25 \mathrm{mg} / \mathrm{ml}$ RhoB dose; $\boldsymbol{B}$, SPG-specific Mdr65-GFP (G $\times$ M/G $\times$ M) overexpression in a WT Mdr65-GFP expression is seen in $\mathrm{G} \times \mathrm{M} / \mathrm{G} \times \mathrm{M}$ double homozygotes over $\mathrm{G} \times \mathrm{M}$ alone (data not shown). Relative RhoB brain fluor content is shown as mean values normalized to WT \pm percentage $S D$ at $4 \mathrm{~h}$ after injection $(n=5-8$ individual brains; $p<$ alone (-) or 3.3 or $6.6 \mathrm{~mm} \mathrm{VB} \mathrm{(+)} \mathrm{and} \mathrm{FITC-} \mathrm{dextran,} \mathrm{respectively.} \mathrm{Sublethal} \mathrm{VB} \mathrm{drug} \mathrm{doses} \mathrm{were} \mathrm{identified} \mathrm{by} \mathrm{assaying} \mathrm{walking}$ behavior after overnight injection (data not shown). Relative brain fluor content is shown as mean values normalized to WT plus representative animals are shown below. Note absolute loss of HEL in c (bottom) when comparing the plus VB condition, $w-W T$ to $P$ Ex8 or diminishing central retinal penetration in $\boldsymbol{d}$ (bottom) when comparing $G$ with $G \times M / G \times M$.

(Dean and Annilo, 2005). To unravel neurochemical protective function, we performed a reverse-genetic, physiologically based screen that takes advantage of large collections of preexisting mutants in many Dm genes and identified PMdr65, a loss-offunction allele of an $\mathrm{ABC} B$ transporter (Fig. 2). To confirm the functional relevance of the PMdr65 mutant to the CNS, we devised additional quantitative and in vivo drug partition assays that address transporter-specific neuroprotective processes (Figs. 3, 4) and localized Mdr65 expression and function to the apical interface of the D $m$ BBB (Fig. 5). Furthermore, human MDR1/Pgp expressed at the Dm BBB could similarly rescue drug transport; thus, MDR1/ Pgp can function cell autonomously to protect a CNS interstitial space. These data show that at least one $\mathrm{ABC}$ transporter in $\mathrm{Dm}$ performs BBB-specific duties similar to vertebrate MDR1/Pgp (Schinkel, 1999) and suggests that the unique demands of CNS chemoprotection may select for transporters that are tuned to neural 
barrier requirements, although very large evolutionary distances obscure the functional relationship of specific genes.

Coincident localization of the diffusion and xenobiotic transport barriers (Fig. 4C) demonstrated that the Dm BBB combines vertebrate-like drug exclusion mechanisms to maintain a chemical barrier for the brain. This is an ideal setting to test the interrelationship of chemical protection components at the cellular, organ, and organismal levels (Strange, 2007). For example, in vertebrates, MDR1/Pgp overexpression specifically promotes chemotheraptic drug resistance in cancer cells (Gottesman et al., 2002). Here the quantity of an individual transporter at the cell membrane can alter the localized pharmacokinetics of a toxin by reducing partition into cells through increased efflux. However, at the BBB, the same gene functions in a complex cellular environment in which xenobiotic protection has the potential to be dependent on not only the content of a single transporter but also cell-type-specific expression, spatial localization in a polarized cellular interface, additional transporters, and other localized pharmacokinetic processes such as diffusion barriers and metabolic enzymes (Sarkadi et al., 2006; Zlokovic, 2008). A strong drug barrier such as the BBB can only function appropriately when all of these properties are manifested and correctly integrated. In this study, we show that increased quantities of BBBspecific Mdr65 induces CNS-specific superprotection to cytotoxic substrates (Fig. 6B). This is dependent on transporter localization, because several biologic tags to Mdr65 that prevent apical membrane association abrogate superprotection and rescue of xenobiotic sensitivity in Mdr65 nulls while exhibiting otherwise normal expression (data not shown). Thus, a single overexpressed transporter can protect both an individual cell and, if properly localized, an entire viscous space such as the CNS from drugs or chemicals. These data support the prevailing paradigm in vertebrate $A B C$ transporter biology that end organ sensitivity must be matched with transporter type, quantity, and discrete localization to promote xenobiotic efflux across cellular interfaces (Sarkadi et al., 2006).

A great advantage of the Drosophila model system is that cellautonomous gain or loss of function can be tested with anatomically directed genetic reagents, an approach that remains a technical challenge in vertebrates. Interestingly, selective, cell-typespecific reduction of Mdr65 in the BBB produces a qualitatively similar xenobiotic phenotype to Mdr65 loss-of-function animals $(\sim 1.7: 1$, mutant to WT) (Fig. $2 E)$; thus, much of the chemoprotective phenotype of Mdr65 is targeted to the SPG. However, this RNAi-induced chemosensitive phenotype is not as strong as Mdr65 loss of function (2.4:1 mutant to WT) (Fig. 2 B), suggesting additional roles for Mdr65 in whole animal small-molecule pharmacokinetics. In fact, anatomically specific gene expression profiling demonstrates heightened levels of Mdr65 at other chemoprotective interfaces such as the gut and malphigian tubules (http://www.flyatlas.org/). Thus, like vertebrate MDR1/Pgp, Mdr65 could play a role in broader xenobiotic/drug physiology, suggesting additional evolutionary conservation between the way vertebrates and invertebrates organize and regulate chemical protection biology.

Finding innovative solutions to the drug delivery problems presented by the $\mathrm{BBB}$, and indeed by all biological barriers, is likely to require an integrated understanding of the physiological mechanisms that allow barriers to maintain a balance between metabolic homeostasis and chemical protection. A genetic system like Dm offers the opportunity to use inducible gene reduction systems and thus gain insight into acute responses to drug efflux loss of function, a condition similar to selectively localizing high levels of a transport inhibitor. Ultimately, these methods may be more gainfully applied to multiple, simultaneous gene reductions at the BBB interface. Such epistasis experiments targeting multiple localized small-molecule partition components will be a powerful method to uncover subtle interactions between localized pharmacokinetic control systems. With these tools in hand, future work will focus on systematic characterization of transport interfaces, through genomics or proteomics, and analysis of barrier responsiveness to various neurologic insults including cytotoxic drugs, hypoxia, and metabolic stress.

Recent analyses of vertebrate barrier components point to a large number of biological pathways that may be involved in controlling and integrating the various aspects of barrier function (Enerson and Drewes, 2006; Zlokovic, 2008) (Ben Barres, personal communication). However, establishing the relevance of potential gene candidates is difficult without model systems that allow rapid analysis of proposed pathway function (Dow, 2007; Yang et al., 2007). We present a framework for using reverse genetics to build an integrated model of BBB physiology and to discover and test regulatory hypotheses of CNS chemoprotection. In addition, this system has shown that simple genetic screens for breakdown of BBB function can identify new and unrecognized genes, like Moody, that are part of the regulatory hierarchy of neuroprotection hinting at modulatable control systems in vertebrate chemoprotection. Indeed, ongoing forward genetic screens have also identified mutants in several $D m$ genes that are highly homologous to relevant vertebrate BBB genes involved in signaling, stress sensing, and establishing and maintaining the structure of the BBB (data not shown). Thus, BBB-specific genes and processes found in model organisms, particularly Drosophila, could lead to novel insights into the organization and cellular separation of the multiple protective BBB physiologies. These considerations, we believe, make our model system remarkably useful in terms of understanding how ancient and resilient organisms, such as the fruit fly, protect their CNS. Last, this approach may promote the identification of common, conserved regulatory pathways that contribute to chemical protection biology and BBB physiology across species.

\section{References}

Abbott NJ (2005) Dynamics of CNS barriers: evolution, differentiation, and modulation. Cell Mol Neurobiol 25:5-23.

Bachmeier CJ, Trickler WJ, Miller DW (2006) Comparison of drug efflux transport kinetics in various blood-brain barrier models. Drug Metab Dispos 34:998-1003.

Bainton RJ, Tsai LT, Schwabe T, DeSalvo M, Gaul U, Heberlein U (2005) moody encodes two GPCRs that regulate cocaine behaviors and bloodbrain barrier permeability in Drosophila. Cell 123:145-156.

Ballabh P, Braun A, Nedergaard M (2004) The blood-brain barrier: an overview: structure, regulation, and clinical implications. Neurobiol Dis $16: 1-13$.

Banerjee S, Sousa AD, Bhat MA (2006) Organization and function of septate junctions: an evolutionary perspective. Cell Biochem Biophys 46:65-77.

Banerjee S, Bainton RJ, Mayer N, Beckstead R, Bhat MA (2008) Septate junctions are required for ommatidial integrity and blood-eye barrier function in Drosophila. Dev Biol 317:585-599.

Bosch I, Jackson GR Jr, Croop JM, Cantiello HF (1996) Expression of Drosophila melanogaster P-glycoproteins is associated with ATP channel activity. Am J Physiol 271:C1527-C1538.

Brand AH, Perrimon N (1993) Targeted gene expression as a means of altering cell fates and generating dominant phenotypes. Development 118:401-415

Calabria AR, Shusta EV (2006) Blood-brain barrier genomics and proteomics: elucidating phenotype, identifying disease targets and enabling brain drug delivery. Drug Discov Today 11:792-799.

Carlson SD, Juang JL, Hilgers SL, Garment MB (2000) Blood barriers of the insect. Annu Rev Entomol 45:151-174. 
Chaudhary PM, Roninson IB (1993) Induction of multidrug resistance in human cells by transient exposure to different chemotherapeutic drugs. J Natl Cancer Inst 85:632-639.

Daneman R, Barres BA (2005) The blood-brain barrier; lessons from moody flies. Cell 123:9-12.

Dean M, Annilo T (2005) Evolution of the ATP-binding cassette (ABC) transporter superfamily in vertebrates. Annu Rev Genomics Hum Genet 6:123-142.

Dean M, Rzhetsky A, Allikmets R (2001) The human ATP-binding cassette (ABC) transporter superfamily. Genome Res 11:1156-1166.

Demeule M, Vachon V, Delisle MC, Beaulieu E, Averill-Bates D, Murphy GF, Béliveau R (1995) Molecular study of P-glycoprotein in multidrug resistance using surface plasmon resonance. Anal Biochem 230:239-247.

Dietzl G, Chen D, Schnorrer F, Su KC, Barinova Y, Fellner M, Gasser B, Kinsey K, Oppel S, Scheiblauer S, Couto A, Marra V, Keleman K, Dickson BJ (2007) A genome-wide transgenic RNAi library for conditional gene inactivation in Drosophila. Nature 448:151-156.

Dohgu S, Yamauchi A, Takata F, Naito M, Tsuruo T, Higuchi S, Sawada Y, Kataoka Y (2004) Transforming growth factor-betal upregulates the tight junction and P-glycoprotein of brain microvascular endothelial cells. Cell Mol Neurobiol 24:491-497.

Dow JA (2007) Integrative physiology, functional genomics and the phenotype gap: a guide for comparative physiologists. J Exp Biol 210:1632-1640.

Edwards JS, Swales LS, Bate M (1993) The differentiation between neuroglia and connective tissue sheath in insect ganglia revisited: the neural lamella and perineurial sheath cells are absent in a mesodermless mutant of Drosophila. J Comp Neurol 333:301-308.

Enerson BE, Drewes LR (2006) The rat blood-brain barrier transcriptome. J Cereb Blood Flow Metab 26:959-973.

Freeman MR, Delrow J, Kim J, Johnson E, Doe CQ (2003) Unwrapping glial biology: Gcm target genes regulating glial development, diversification, and function. Neuron 38:567-580.

Garberg P, Ball M, Borg N, Cecchelli R, Fenart L, Hurst RD, Lindmark T, Mabondzo A, Nilsson JE, Raub TJ, Stanimirovic D, Terasaki T, Oberg JO, Osterberg T (2005) In vitro models for the blood-brain barrier. Toxicol In Vitro 19:299-334.

Gerrard B, Stewart C, Dean M (1993) Analysis of Mdr50: a Drosophila Pglycoprotein/multidrug resistance gene homolog. Genomics 17:83-88.

Gottesman MM, Fojo T, Bates SE (2002) Multidrug resistance in cancer: role of ATP-dependent transporters. Nat Rev Cancer 2:48-58.

Kartner N, Evernden-Porelle D, Bradley G, Ling V (1985) Detection of P-glycoprotein in multidrug-resistant cell lines by monoclonal antibodies. Nature 316:820-823.

Löscher W, Potschka H (2005a) Role of drug efflux transporters in the brain for drug disposition and treatment of brain diseases. Prog Neurobiol 76:22-76.

Löscher W, Potschka H (2005b) Blood-brain barrier active efflux transporters: ATP-binding cassette gene family. NeuroRx 2:86-98.

Nag S (2003) The blood-brain barrier. Totowa, NJ: Humana.

Neuwelt E, Abbott NJ, Abrey L, Banks WA, Blakley B, Davis T, Engelhardt B, Grammas P, Nedergaard M, Nutt J, Pardridge W, Rosenberg GA, Smith Q, Drewes LR (2008) Strategies to advance translational research into brain barriers. Lancet Neurol 7:84-96.
Nitta T, Hata M, Gotoh S, Seo Y, Sasaki H, Hashimoto N, Furuse M, Tsukita S (2003) Size-selective loosening of the blood-brain barrier in claudin5-deficient mice. J Cell Biol 161:653-660.

Pardridge WM (2005a) The blood-brain barrier: bottleneck in brain drug development. NeuroRx 2:3-14.

Pardridge WM (2005b) Molecular biology of the blood-brain barrier. Mol Biotechnol 30:57-70.

Pereanu W, Shy D, Hartenstein V (2005) Morphogenesis and proliferation of the larval brain glia in Drosophila. Dev Biol 283:191-203.

Riordan JR, Deuchars K, Kartner N, Alon N, Trent J, Ling V (1985) Amplification of P-glycoprotein genes in multidrug-resistant mammalian cell lines. Nature 316:817-819.

Sarkadi B, Homolya L, Szakács G, Váradi A (2006) Human multidrug resistance $\mathrm{ABCB}$ and $\mathrm{ABCG}$ transporters: participation in a chemoimmunity defense system. Physiol Rev 86:1179-1236.

Schinkel AH (1997) The physiological function of drug-transporting P-glycoproteins. Semin Cancer Biol 8:161-170.

Schinkel AH (1999) P-Glycoprotein, a gatekeeper in the blood-brain barrier. Adv Drug Deliv Rev 36:179-194.

Schinkel AH, Mayer U, Wagenaar E, Mol CA, van Deemter L, Smit JJ, van der Valk MA, Voordouw AC, Spits H, van Tellingen O, Zijlmans JM, Fibbe WE, Borst P (1997) Normal viability and altered pharmacokinetics in mice lacking mdrl-type (drug-transporting) P-glycoproteins. Proc Natl Acad Sci U S A 94:4028-4033.

Schwabe T, Bainton RJ, Fetter RD, Heberlein U, Gaul U (2005) GPCR signaling is required for blood-brain barrier formation in drosophila. Cell 123:133-144.

Stork T, Engelen D, Krudewig A, Silies M, Bainton RJ, Klämbt C (2008) Organization and function of the blood-brain barrier in Drosophila J Neurosci 28:587-597.

Strange K (2007) Revisiting the Krogh Principle in the post-genome era: Caenorhabditis elegans as a model system for integrative physiology research. J Exp Biol 210:1622-1631.

Sullivan W, Ashburner M, Hawley RS (2000) Drosophila protocols. Cold Spring Harbor, NY: Cold Spring Harbor Laboratory.

Tepass U, Hartenstein V (1994) The development of cellular junctions in the Drosophila embryo. Dev Biol 161:563-596.

Treherne JE, Pichon Y (1972) The insect blood-brain barrier. London: Academic.

van Den Elsen JM, Kuntz DA, Hoedemaeker FJ, Rose DR (1999) Antibody C219 recognizes an alpha-helical epitope on P-glycoprotein. Proc Natl Acad Sci U S A 96:13679-13684.

Wu CT, Budding M, Griffin MS, Croop JM (1991) Isolation and characterization of Drosophila multidrug resistance gene homologs. Mol Cell Biol 11:3940-3948.

Wu VM, Beitel GJ (2004) A junctional problem of apical proportions: epithelial tube-size control by septate junctions in the Drosophila tracheal system. Curr Opin Cell Biol 16:493-499.

Yang J, McCart C, Woods DJ, Terhzaz S, Greenwood KG, ffrench-Constant RH, Dow JA (2007) A Drosophila systems approach to xenobiotic metabolism. Physiol Genomics 30:223-231.

Zlokovic BV (2008) The blood-brain barrier in health and chronic neurodegenerative disorders. Neuron 57:178-201. 